\title{
The Tate conjecture for certain abelian varieties over finite fields
}

\author{
by \\ J. S. Milne (Ann Arbor, MI)
}

In an earlier work, we showed that if the Hodge conjecture holds for all complex abelian varieties of CM-type, then the Tate conjecture holds for all abelian varieties over finite fields (Milne 1999b). In this article, we extract from the proof a statement (Theorem 1.1) that sometimes allows one to deduce the Tate conjecture for the powers of a single abelian variety $A$ over a finite field from knowing that some Hodge classes on their lifts to characteristic zero are algebraic.

Tate's theorem (Tate 1966) implies that the Tate conjecture holds for any abelian variety over a finite field whose $\mathbb{Q}_{\ell}$-algebra of Tate classes is generated by those of degree 1 . Examples are known of abelian varieties for which this condition (and hence the Tate conjecture) hold (Lenstra, Spiess, Zarhin; see the examples in A.7 below). Using Theorem 1.1 and a result of Schoen $(1988,1998)$, we construct examples of abelian varieties for which the condition fails, but for which we are nevertheless able to prove the Tate conjecture (see 1.7, 1.8).

The main results are stated in Section 1 and proved in Section 2. Appendix A summarizes the theories of abelian varieties of CM-type over $\mathbb{C}$ and of abelian varieties over finite fields, and how the reduction map relates the two. Appendix B sharpens a result of Clozel on the relation between numerical and homological equivalence for abelian varieties over finite fields.

Notations not introduced in Section 1 are listed at the start of Appendix A.

1. Statements. Let $X$ be a smooth complete variety over an algebraic closure $\mathbb{F}$ of the field $\mathbb{F}_{p}$ of $p$ elements. The choice of a model $X_{1}$ of $X$ over a subfield $\mathbb{F}_{p^{n}}$ of $\mathbb{F}$ determines an action of $\operatorname{Gal}\left(\mathbb{F} / \mathbb{F}_{p^{n}}\right)$ on the étale cohomology group $H^{2 r}\left(X, \mathbb{Q}_{\ell}(r)\right)$, and we define

2000 Mathematics Subject Classification: 11G10, 14K15, 14C25.

This article includes research supported in part by the National Science Foundation. 


$$
\mathcal{T}_{\ell}^{r}(X)=\bigcup_{X_{1} / \mathbb{F}_{p^{n}}} H^{2 r}\left(X, \mathbb{Q}_{\ell}(r)\right)^{\operatorname{Gal}\left(\mathbb{F} / \mathbb{F}_{p^{n}}\right)}
$$

(union over all models). The elements of $\mathcal{T}_{\ell}^{r}(X)$ are called the $\ell$-adic Tate classes of degree $r$ on $X$. We shall say that the Tate conjecture holds for $X$ if the $\mathbb{Q}_{\ell}$-vector space $\mathcal{T}_{\ell}^{r}(X)$ is spanned by the classes of algebraic cycles for all $r$ and all $\ell \neq p$.

A Tate class is said to be exotic if it is not in the $\mathbb{Q}_{\ell}$-algebra generated by the Tate classes of degree 1 . For an abelian variety over $\mathbb{F}$, Tate (1966) showed that all Tate classes of degree 1 are divisor classes, and so the nonexotic Tate classes are algebraic.

Let $A_{0}$ be an abelian variety over $\mathbb{F}$. If the Tate conjecture holds for $A_{0}$, then the equivalent statements of Tate 1994, Theorem 2.9, hold for every model $A_{1}$ of $A$ over a finite field. In particular, the Tate conjecture holds for $A_{1} / \mathbb{F}_{q}$, and for every $r$, the order of the pole of the zeta function $Z\left(A_{1}, t\right)$ of $A_{1}$ at $t=q^{-r}$ is equal to the rank of the group of numerical equivalence classes of algebraic cycles of codimension $r$ on $A_{1}$.

Let $A$ be an abelian variety with many endomorphisms (see A.2) over an algebraically closed field $k$. Then (see A.3) there is a group of multiplicative type $L(A)$ over $\mathbb{Q}$ whose fixed tensors in any Weil cohomology of a power $A^{s}$ of $A$ are exactly the Lefschetz classes, i.e., those in the algebra generated by divisor classes. We call $L(A)$ the Lefschetz group of $A$.

Now take $k$ to be the algebraic closure $\mathbb{Q}^{\text {al }}$ of $\mathbb{Q}$ in $\mathbb{C}$, and let $w_{0}$ be a prime of $\mathbb{Q}^{\text {al }}$ dividing $p$. It follows from the theory of Néron models, that $A$ has good reduction at $w_{0}$ (Serre and Tate 1968, Theorem 6) and so defines an abelian variety $A_{0}$ over the residue field $\mathbb{F}$ at $w$. There is a canonical inclusion $L\left(A_{0}\right) \hookrightarrow L(A)$ (see A.3, Remark).

Let $H^{r}(A, \mathbb{Q})$ denote the usual cohomology group of the complex manifold $A(\mathbb{C})$, and let $H^{r}(A, \mathbb{Q}(m))=H^{r}\left(A,(2 \pi i)^{m} \mathbb{Q}\right)$ - it is a rational Hodge structure of weight $r-2 m$. The action of $L(A)$ on $H^{2 r}\left(A^{s}, \mathbb{Q}(r)\right)$ defines a decomposition

$$
H^{2 r}\left(A^{s}, \mathbb{Q}(r)\right) \otimes \mathbb{Q}^{\mathrm{al}}=\bigoplus_{\chi \in X^{*}(L(A))} H^{2 r}\left(A^{s}, \mathbb{Q}(r)\right)_{\chi}
$$

where $(-)_{\chi}$ is the subspace on which $L(A)$ acts through its character $\chi$. We say that $\chi$ is algebraic if $H^{2 r}\left(A^{s}, \mathbb{Q}(r)\right)_{\chi}$ contains a nonzero algebraic class for some $r$ and $s$. The set of algebraic characters of $L(A)$ is stable under the action of $\operatorname{Gal}\left(\mathbb{Q}^{\text {al }} / \mathbb{Q}\right)$, and if $\chi$ is algebraic then $H^{2 r}\left(A^{s}, \mathbb{Q}(r)\right)_{\chi}$ consists entirely of algebraic classes $\left({ }^{1}\right)$. By composition, an algebraic character of $L(A)$ defines a character of $L\left(A_{0}\right)$.

$\left({ }^{1}\right)$ The algebraic characters are precisely those that are trivial on the subgroup $M(A)$ of $L(A)$ (see A.3). 
A model $A_{1} / \mathbb{F}_{q}$ of $A_{0}$ over a finite field defines a Frobenius endomorphism $\pi$ of $A_{0}$. Some power of $\pi$ lies in $L\left(A_{0}\right)(\mathbb{Q})$, and we define $P\left(A_{0}\right)$ to be the smallest algebraic subgroup of $L\left(A_{0}\right)$ containing a power of $\pi$ (see A.3).

THEOREM 1.1. If

$$
P\left(A_{0}\right)=\bigcap \operatorname{Ker}\left(\chi: L\left(A_{0}\right) \rightarrow \mathbb{G}_{m}\right)
$$

(intersection over the algebraic characters of $L(A)$ ), then the Tate conjecture holds for all powers of $A_{0}$.

An element of $H^{2 r}(A, \mathbb{Q}(r)) \cap H^{0,0}$ is called a Hodge class of degree $r$ on $A$. We say that the Hodge conjecture holds for $A$ if the $\mathbb{Q}$-vector space of Hodge classes on $A$ of degree $r$ is spanned by the classes of algebraic cycles for all $r$. The Mumford-Tate group $\mathrm{MT}(A)$ of $A$ is the largest algebraic subgroup of $L(A)$ fixing the Hodge classes on all powers of $A$.

Corollary 1.2. If the Hodge conjecture holds for all powers of $A$ and

$$
\left.P\left(A_{0}\right)=L\left(A_{0}\right) \cap \mathrm{MT}(A) \quad \text { (intersection inside } L(A)\right),
$$

then the Tate conjecture holds for all powers of $A_{0}$.

A Hodge class is said $\left({ }^{2}\right)$ to be exotic if it is not in the $\mathbb{Q}$-algebra generated by Hodge classes of degree 1. Lefschetz showed that all Hodge classes of degree 1 are divisor classes, and so the nonexotic Hodge classes are exactly the Lefschetz classes (in particular, they are algebraic).

Let $E$ be a CM-field of degree $2 n, n>2$, over $\mathbb{Q}$ containing a quadratic imaginary field $Q$. Choose an embedding $\varrho_{0}: Q \rightarrow \mathbb{Q}^{\text {al }}$, and let $\left\{\sigma_{0}, \ldots, \sigma_{n-1}\right\}$ be the set of extensions of $\varrho_{0}$ to $E$. Then $\Phi_{0}={ }_{\mathrm{df}}\left\{\sigma_{0}, \iota \circ \sigma_{1}, \ldots, \iota \circ \sigma_{n-1}\right\}$ ( $\iota$ denotes complex conjugation on $\mathbb{C}$ ) is a CM-type on $E$ and $\Phi={ }_{\mathrm{df}}\left\{\varrho_{0}\right\}$ is a CM-type on $Q$. Let $(A, i)$ and $(B, j)$ be abelian varieties over $\mathbb{Q}^{\text {al }}$ of CMtypes $\left(E, \Phi_{0}\right)$ and $(Q, \Phi)$ respectively. We let $Q$ act diagonally on $A \times B^{n-2}$.

Lemma 1.3. The exotic Hodge classes on $A \times B^{n-2}$ are exactly the nonzero elements of the subspace

$$
W(A, B) \stackrel{\mathrm{df}}{=}\left(\bigwedge_{Q}^{2 n-2} H^{1}\left(A \times B^{n-2}, \mathbb{Q}\right)\right)(n-1)
$$

of $H^{2 n-2}\left(A \times B^{n-2}, \mathbb{Q}(n-1)\right)$.

As $A \times B^{n-2}$ has dimension $2 n-2, H^{1}\left(A \times B^{n-2}, \mathbb{Q}\right)$ has dimension $4 n-4$ over $\mathbb{Q}$, and so $\bigwedge_{Q}^{2 n-2} H^{1}\left(A \times B^{n-2}, \mathbb{Q}\right)$ has dimension 1 over $Q$. The action of an endomorphism of an abelian variety on its cohomology groups preserves algebraic classes, and so, if $W(A, B)$ contains one nonzero algebraic class, then it is spanned as a $\mathbb{Q}$-space by algebraic classes.

$\left({ }^{2}\right)$ Following Tate 1994, p. 82. 
THEOREM 1.4. If some exotic Hodge class on $A \times B^{n-2}$ is algebraic, then the Hodge conjecture holds for all abelian varieties of the form $A^{s} \times B^{t}$, $s, t \in \mathbb{N}$.

The abelian varieties $A$ and $B$ over $\mathbb{Q}^{\text {al }}$ reduce modulo $w_{0}$ to abelian varieties $A_{0}$ and $B_{0}$ over $\mathbb{F}$. Let $K$ be the Galois closure of $\sigma_{0} E$ in $\mathbb{Q}^{\text {al }}$, and let $D\left(w_{0}\right)$ be the decomposition group of $w_{0}$ in $\operatorname{Gal}(K / \mathbb{Q})$.

TheOrem 1.5. Assume $p$ splits in $Q$ and that $\operatorname{Gal}\left(K / \sigma_{0} E\right) \cdot D\left(w_{0}\right)$ is a subgroup of $\operatorname{Gal}(K / \mathbb{Q})$.

(a) For all $\ell \neq p$, the exotic $\ell$-adic Tate classes on $A_{0} \times B_{0}^{n-2}$ are exactly the nonzero elements of the subspace

$$
W\left(A_{0}, B_{0}\right) \stackrel{\text { df }}{=}\left(\bigwedge_{Q \otimes \mathbb{Q} \mathbb{Q}_{\ell}}^{2 n-2} H^{1}\left(A_{0} \times B_{0}^{n-2}, \mathbb{Q}_{\ell}\right)\right)(n-1)
$$

of $H^{2 n-2}\left(A_{0} \times B_{0}^{n-2}, \mathbb{Q}_{\ell}(n-1)\right)$.

(b) If some exotic Hodge class on $A \times B^{n-2}$ is algebraic, then the Tate conjecture holds for all abelian varieties over $\mathbb{F}$ of the form $A_{0}^{s} \times B_{0}^{t}, s, t \in \mathbb{N}$.

REMARK 1.6. (a) Note that, under the hypotheses of the theorem, the $\mathbb{Q}$-algebra of Hodge classes on $A \times B^{n-2}$ is larger than the tensor product of the similar algebras for $A$ and $B^{n-2}$, and the $\mathbb{Q}_{\ell}$-algebra of Tate classes on $A_{0} \times B_{0}^{n-2}$ is larger than the tensor product of the similar algebras for $A_{0}$ and $B_{0}^{n-2}$. Moreover, the groups $L(A \times B)$ and $\mathrm{MT}(A \times B)\left(\right.$ resp. $L\left(A_{0} \times B_{0}\right)$ and $\left.P\left(A_{0} \times B_{0}\right)\right)$ are not distinguished by their fixed tensors in the cohomology of $A \times B$ (resp. $\left.A_{0} \times B_{0}\right)$.

(b) The condition that $\operatorname{Gal}\left(K / \sigma_{0} E\right) \cdot D\left(w_{0}\right)$ be a subgroup of $\operatorname{Gal}(K / \mathbb{Q})$ holds, for example, if $E$ is Galois over $\mathbb{Q}$. Without it, the analysis becomes very complicated, and the theorem fails.

Examples. Let $C$ be an abelian variety over $\mathbb{C}$, and let $i: Q \rightarrow \operatorname{End}^{0}(C)$ be a homomorphism of $\mathbb{Q}$-algebras, where, as above, $Q$ is a quadratic imaginary extension of $\mathbb{Q}$. The pair $(C, i)$ is said to be of Weil type if the tangent space to $C$ at 0 is a free $Q \otimes_{\mathbb{Q}} \mathbb{C}$-module.

When $(C, i)$ is of Weil type, its dimension is even, say, $\operatorname{dim} C=2 m$, and the subspace $\left(\bigwedge_{Q}^{2 m} H^{1}(C, \mathbb{Q})\right)(m)$ of $H^{2 m}(C, \mathbb{Q}(m))$ consists of Hodge classes (Weil 1977) - they are called the Weil classes on $C$.

Let $\lambda$ be a polarization of $C$ whose Rosati involution induces complex conjugation on $Q$, and let $E^{\lambda}$ be the Riemann form defined by $\lambda$. There exists a skew-Hermitian form $\phi: H_{1}(A, \mathbb{Q}) \times H_{1}(A, \mathbb{Q}) \rightarrow Q$ such that $\operatorname{Tr}_{Q / \mathbb{Q}} \circ \phi=E$. The discriminant of $\phi$ is an element of $\mathbb{Q}^{\times} / \mathrm{Nm}\left(Q^{\times}\right)$which is independent of the choice of the polarization, and so can be denoted by $\operatorname{det}(C, i)$. The quotient $\mathbb{Q}^{\times} / \operatorname{Nm}\left(Q^{\times}\right)$is an infinite group killed by 2 , and for any $a \in \mathbb{Q}^{\times} / \operatorname{Nm}\left(Q^{\times}\right)$with $(-1)^{m} a>0$, there exists an $m^{2}$-dimensional family of abelian varieties of Weil type with determinant $a$ (Weil 1977, van 
Geemen 1994). We say that $\phi$ is split when there is an $m$-dimensional $Q$ subspace of $H_{1}(A, \mathbb{Q})$ on which $\phi$ is totally isotropic.

The Weil classes on $C$ are known to be algebraic in the following cases:

(a) $Q=\mathbb{Q}[\sqrt{-3}], m=3$, and $\phi$ is split (Schoen 1998; see also van Geemen 1994, 7.3, p. 250);

(b) $Q=\mathbb{Q}[\sqrt{-3}], m=2$ (Schoen 1988 when $\phi$ is split and Schoen 1998 in general);

(c) $Q=\mathbb{Q}[\sqrt{-1}], m=2$, and $\phi$ is split (van Geemen 1996).

Corollary 1.7. Let $A, B, E, Q$ be as in Theorem 1.5, and let $Q$ act diagonally on $A \times B^{n-2}$. If the Weil classes on $A \times B^{n-2}$ are algebraic, then the Hodge conjecture holds for all abelian varieties of the form $A^{s} \times B^{t}$, $s, t \in \mathbb{N} \times \mathbb{N}$, and the Tate conjecture holds for all abelian varieties of the form $A_{0}^{s} \times B_{0}^{t}, s, t \in \mathbb{N} \times \mathbb{N}$.

Proof. In this case, $W(A, B)$ is the space of Weil classes on $A \times B^{n-2}$.

EXAmPle 1.8. Let $Q=\mathbb{Q}[\sqrt{-3}]$ and let $p$ be a prime such that $p \equiv 1$ $(\bmod 3)$; let $F$ be a totally real cubic extension of $\mathbb{Q}$ that is Galois over $\mathbb{Q}$ or such that $p$ splits in it, and let $E=F \cdot Q$; let $\Phi=\left\{\varrho_{0}\right\}$ and $\Phi_{0}=\left\{\sigma_{0}, \iota \sigma_{1}, \iota \sigma_{2}\right\}$ be the CM-types on $Q$ and $E$ respectively defined above. Then, for all abelian 3 -folds $A$ of CM-type $\left(E, \Phi_{0}\right)$ and all elliptic curves $B$ of CM-type $(Q, \Phi)$,

(a) the Hodge conjecture holds for the abelian varieties $A^{s} \times B^{t}, s, t \in \mathbb{N}$; the subspace $W(A, B)$ of $H^{4}(A \times B, \mathbb{Q}(2))$ consists of exotic Hodge classes;

(b) the Tate conjecture holds for the abelian varieties $A_{0}^{s} \times B_{0}^{t}, s, t \in \mathbb{N}$; the subspace $W\left(A_{0}, B_{0}\right)$ of $H^{4}\left(A_{0} \times B_{0}, \mathbb{Q}_{\ell}(2)\right)$ consists of exotic Tate classes.

2. Proofs. Notations concerning groups of multiplicative type are reviewed at the start of Appendix A.

Proof of 1.1. After the Theorem in A.3, in order to prove Theorem 1.1, it suffices to show that its hypotheses imply that $M\left(A_{0}\right)=P\left(A_{0}\right)$.

As numerical equivalence agrees with homological equivalence on abelian varieties in characteristic zero (see B.1), we may regard $M(A)$ as the subgroup of $L(A)$ fixing the algebraic classes in $H^{2 r}\left(A^{s}, \mathbb{Q}(r)\right)$ for all $r$, s, i.e., as the intersection of the kernels of the algebraic characters on $L(A)$. Hence

$$
L\left(A_{0}\right) \cap M(A)=\bigcap_{\chi \text { algebraic }} \operatorname{Ker}\left(\chi: L\left(A_{0}\right) \rightarrow \mathbb{G}_{m}\right) .
$$

Thus, the hypotheses of Theorem 1.1 imply that $L\left(A_{0}\right) \cap M(A)=P\left(A_{0}\right)$. Since

$$
L\left(A_{0}\right) \cap M(A) \supset M\left(A_{0}\right) \supset P\left(A_{0}\right),
$$

this implies that $M\left(A_{0}\right)=P\left(A_{0}\right)$. 
Proof of 1.2. As we noted in the proof of 1.1,

$$
M(A)=\bigcap_{\chi \text { algebraic }} \operatorname{Ker}\left(\chi: L(A) \rightarrow \mathbb{G}_{m}\right) .
$$

If the Hodge conjecture holds for the powers of $A$, then $\operatorname{MT}(A)=M(A)$ (see A.3). If, in addition, $P\left(A_{0}\right)=L\left(A_{0}\right) \cap \mathrm{MT}(A)$, then

$$
P\left(A_{0}\right)=\bigcap_{\chi} \operatorname{Ker}\left(\chi: L\left(A_{0}\right) \rightarrow \mathbb{G}_{m}\right)
$$

(intersection over the algebraic characters of $L(A)$ ), and so (1.2) follows from (1.1).

Proofs of 1.3 and 1.4. Let $E, Q, \varrho_{0},\left\{\sigma_{0}, \ldots, \sigma_{n-1}\right\}, \Phi$ and $\Phi_{0}$ be as in the paragraph preceding the statement of Lemma 1.3. Let $K$ be a CM-subfield of $\mathbb{Q}^{\text {al }}$, finite and Galois over $\mathbb{Q}$, containing the Galois closure of $\sigma_{0} E$ in $\mathbb{Q}^{\text {al }}$, and let $S^{K}$ be its Serre group (see A.4). For each $i, 0 \leq i \leq n-1$, let

$$
\Sigma_{i}=\left\{\tau \in \operatorname{Gal}(K / \mathbb{Q}) \mid \tau \circ \sigma_{0}=\sigma_{i}\right\} .
$$

Then $\Sigma_{0}$ is the subgroup $\operatorname{Gal}\left(K / \sigma_{0} E\right)$ of $\operatorname{Gal}(K / \mathbb{Q})$ and $\Sigma_{0}, \ldots, \Sigma_{n-1}, \iota \Sigma_{0}$, $\ldots, \iota \Sigma_{n-1}$ are its left cosets. Let $\psi_{i}$ be the characteristic function of $\Sigma_{i} \cup$ $\bigcup_{j \neq i} \iota \Sigma_{j}$, and let $\psi$ be the characteristic function of $\bigcup \Sigma_{i}=\left\{\tau \mid \tau \circ \varrho_{0}=\varrho_{0}\right\}$. Note that $\Sigma_{K}$ acts on the set $\left\{\Sigma_{0}, \ldots, \iota \Sigma_{n-1}\right\}$, and that if $\tau \Sigma_{i}=\Sigma_{i^{\prime}}$, then $\tau \psi_{i}=\psi_{i^{\prime}}$. The linear relations among $\psi_{0}, \ldots, \psi_{n-1}, \psi, \iota \psi$ regarded as elements of $X^{*}\left(S^{K}\right)$ are exactly the multiples of

$$
\psi_{0}+\ldots+\psi_{n-1}+(n-2) \psi=(n-1)(\psi+\iota \psi) .
$$

Let $(A, i)$ be an abelian variety of CM-type $\left(E, \Phi_{0}\right)$, and let $X^{*}(L(A))$ be identified with a quotient of $\mathbb{Z}^{\Sigma_{E}}$ (see A.5). The map

$$
X^{*}\left(\varrho_{\Phi_{0}}\right): X^{*}(L(A)) \rightarrow X^{*}\left(S^{K}\right)
$$

(ibid.) sends $\left[\sigma_{0}\right]$ to $\psi_{0}$ and hence, by equivariance and linearity, it sends $\left[\sigma_{i}\right]$ to $\psi_{i}$ and $\left[\sigma_{0}+\iota \sigma_{0}\right]$ to $\psi_{0}+\iota \psi_{0}=\psi+\iota \psi$. Because $\left[\sigma_{0}\right], \ldots,\left[\sigma_{n-1}\right],\left[\sigma_{0}+\iota \sigma_{0}\right]$ form a basis for $X^{*}(L(A))$ and $\psi_{0}, \ldots, \psi_{n-1}, \psi+\iota \psi$ are linearly independent in $X^{*}\left(S^{K}\right)$, we see that $X^{*}\left(\varrho_{\Phi_{0}}\right): X^{*}(L(A)) \rightarrow X^{*}\left(S^{K}\right)$ is injective. Therefore, $\varrho_{\Phi_{0}}: S^{K} \rightarrow L(A)$ is surjective, and $\operatorname{MT}(A)=L(A)$ (ibid.). Hence all Hodge classes on all powers of $A$ are Lefschetz (A.3, Theorem). In particular, the Hodge conjecture holds for $A$ and its powers.

Let $(B, i)$ be an elliptic curve of CM-type $(Q, \Phi)$. In this case, $X^{*}(L(B))$ $=\mathbb{Z}^{\Sigma_{Q}}$ and $L(B)=\left(\mathbb{G}_{m}\right)_{Q / \mathbb{Q}}$. The map $X^{*}\left(\varrho_{\Phi}\right)$ sends $\varrho_{0}$ to $\psi$ and $\iota \varrho_{0}$ to $\iota \psi$. As $\psi$ and $\iota \psi$ are linearly independent in $X\left(S^{K}\right)$, this shows that $\operatorname{MT}(B)=L(B)$, and so all Hodge classes on all powers of $B$ are Lefschetz.

The abelian variety $A$ is simple because its CM-type is primitive (this uses that $n>2)$. The product $A \times B$ is of CM-type $\left(E \times Q, \Phi^{\prime}\right)$ where 
$\Phi^{\prime}=\Phi_{0} \sqcup \Phi$. The group $X^{*}(L(A \times B))$, regarded as a quotient of $\mathbb{Z}^{\Sigma_{E} \sqcup \Sigma_{Q}}$, has basis $\left\{\left[\sigma_{0}\right], \ldots,\left[\sigma_{n-1}\right],\left[\varrho_{0}\right],\left[\varrho_{0}+\iota \varrho_{0}\right]\right\}$, and $X^{*}\left(\varrho_{\Phi^{\prime}}\right)$ sends

$$
\left[\sigma_{i}\right] \mapsto \psi_{i}, \quad\left[\varrho_{0}\right] \mapsto \psi, \quad\left[\varrho_{0}+\iota \varrho_{0}\right] \mapsto \psi+\iota \psi .
$$

Since $(*)$ is the only relation among $\psi_{0}, \ldots, \psi_{n-1}, \psi, \iota \psi$, the kernel of $X^{*}(L(A \times B)) \rightarrow X^{*}\left(S^{K}\right)$ is free of rank 1 with generator

$$
\chi=\left[\sigma_{0}+\ldots+\sigma_{n-1}+(n-2) \varrho_{0}-(n-1)\left(\varrho_{0}+\iota \varrho_{0}\right)\right] .
$$

As $\operatorname{MT}(A \times B)$ is the image of $S^{K}$ in $L(A \times B)$ (see A.5), this shows that there is an exact sequence

$$
0 \rightarrow \mathrm{MT}(A \times B) \rightarrow L(A \times B) \stackrel{\chi}{\rightarrow} T \rightarrow 0
$$

where $T$ is the 1 -dimensional torus over $\mathbb{Q}$ whose character group $\langle\chi\rangle$ is isomorphic to $\mathbb{Z}$ with $\operatorname{Gal}(K / \mathbb{Q})$ acting nontrivially through $\operatorname{Gal}\left(\varrho_{0} Q / \mathbb{Q}\right)$.

The exotic Hodge classes on $A \times B$ and its powers are those that lie in a rational subspace on which $L(A \times B)$ acts through the characters $m \chi$, $m \neq 0$.

We now prove 1.3. The Lefschetz group of $A \times B^{n-2}$ equals that of $A \times B$. It acts on

$$
W(A, B)=\bigwedge_{Q}^{n} H^{1}(A) \otimes \bigwedge_{Q}^{n-2}\left((n-2) H^{1}(B)\right) \otimes \mathbb{Q}(n-1)
$$

through the characters $\chi$ and $\iota \chi=-\chi$. Because $\chi$ is trivial on $\operatorname{MT}(A \times B)$, this space consists of Hodge classes, and because $\chi$ is not trivial on $L(A \times B)$, the Hodge classes are exotic. The group $L(A \times B)$ acts on no other subspace of a space $H^{2 r}\left(A \times B^{n-2}, \mathbb{Q}(r)\right) \otimes \mathbb{Q}^{\text {al }}$ through the characters $\pm \chi$, and so the elements of $W(A, B)$ are the only exotic Hodge classes on $A \times B^{n-2}$.

We now prove 1.4. If some exotic Hodge class in $A \times B^{n-2}$ is algebraic, then the character $\chi$ is trivial on $M(A \times B)$. Hence $M(A \times B)=\mathrm{MT}(A \times B)$. But $M\left(A^{s} \times B^{t}\right)=M(A \times B)$ and $\operatorname{MT}\left(A^{s} \times B^{t}\right)=\mathrm{MT}(A \times B)$ for any $s, t \geq 1$ (see A.5), and so the Hodge conjecture holds for $A^{s} \times B^{t}$ (see A.3).

Proof of 1.5. We shall compute the terms in the diagram

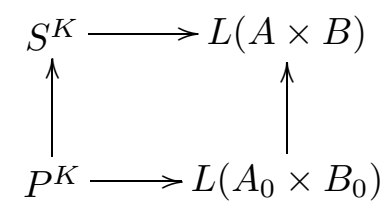

or, equivalently, in the corresponding diagram of character groups. In fact, 
we shall prove that there is an exact commutative diagram

$(* *)$

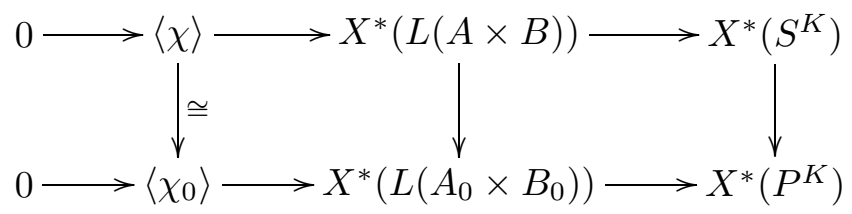

The horizontal maps in the right-hand square are those defined in A.5 and A.7, the map $X^{*}\left(S^{K}\right) \rightarrow X^{*}\left(P^{K}\right)$ is that in the fundamental diagram (A.8), and the map $L\left(A_{0} \times B_{0}\right) \rightarrow L(A \times B)$ comes from the inclusion $C\left(A_{0} \times B_{0}\right) \subset$ $C(A \times B)$ induced by the reduction map $\operatorname{End}^{0}(A \times B) \hookrightarrow \operatorname{End}^{0}\left(A_{0} \times B_{0}\right)$. The character $\chi$ of $L(A \times B)$ is that defined above, and $\chi_{0}$ is the composite of $\chi$ with $L\left(A_{0} \times B_{0}\right) \rightarrow L(A \times B)$.

The "subgroup" condition in the statement of the theorem implies that $\Sigma_{0} \cdot D\left(w_{0}\right)$ is a subgroup of $\operatorname{Gal}(K / \mathbb{Q})$, even though we are no longer assuming $K$ to be the Galois closure of $\sigma_{0} E$. In fact, we now assume that $K$ is large enough to split $\operatorname{End}^{0}\left(A_{0} \times B_{0}\right)$ (in the sense of A.6).

Let $X$ be the set of primes of $K$ dividing $p$. Suppose that the subsets $\Sigma_{i} \cdot w_{0}$ and $\Sigma_{j} \cdot w_{0}$ of $X$ have nonempty intersection. Then $\tau_{i} w_{0}=\tau_{j} w_{0}$ for some $\tau_{i} \in \Sigma_{i}$ and $\tau_{j} \in \Sigma_{j}$. Hence $\tau_{i} \in \tau_{j} D\left(w_{0}\right)$, and so

$$
\Sigma_{i} \cdot w_{0}=\tau_{i} \Sigma_{0} \cdot w_{0} \subset \tau_{j} D\left(w_{0}\right) \Sigma_{0} \cdot w_{0}=\tau_{j} \Sigma_{0} D\left(w_{0}\right) \cdot w_{0}=\Sigma_{j} \cdot w_{0} .
$$

By symmetry, $\Sigma_{i} \cdot w_{0} \supset \Sigma_{j} \cdot w_{0}$, and so the two sets are equal: we have shown that the sets $\Sigma_{i} \cdot w_{0}$ and their complex conjugates form a partition of $X$. Let $X_{0}, \ldots, X_{m-1}$ be the distinct elements of $\left\{\Sigma_{i} \cdot w_{0} \mid 0 \leq i \leq n-1\right\}$ with $X_{0}$ chosen to be $\Sigma_{0} w_{0}$, and let

$$
Y=\left\{X_{0}, \ldots, X_{m-1}, \iota X_{0}, \ldots, \iota X_{m-1}\right\} .
$$

The group $\Sigma_{K}$ acts transitively on $X$ and $Y$, and the stabilizers of $w_{0}$ and $X_{0}$ are $D\left(w_{0}\right)$ and $\Sigma_{0} \cdot D\left(w_{0}\right)$ respectively. By using $w_{0}$ and $X_{0}$ as base points, we can identify the map of $\Sigma_{K}$-sets $X \rightarrow Y$ with $\Sigma_{K} / D\left(w_{0}\right) \rightarrow \Sigma_{K} / \Sigma_{0} \cdot D\left(w_{0}\right)$. Each $X_{j}$ then corresponds to the quotient of a left coset of $\Sigma_{0} \cdot D\left(w_{0}\right)$ by the right action of $D\left(w_{0}\right)$. From these remarks, we see that

$$
\begin{aligned}
|X| & =\left(\Sigma_{K}: D\left(w_{0}\right)\right), \\
|Y| & =\left(\Sigma_{K}: \Sigma_{0} \cdot D\left(w_{0}\right)\right) \quad(=2 m), \\
\left|X_{j}\right| & =\left(\Sigma_{0} \cdot D\left(w_{0}\right): D\left(w_{0}\right)\right) .
\end{aligned}
$$

For $i \in\{0, \ldots, n-1\}$, define $j(i)$ to be the element of $\{0, \ldots, m-1\}$ such that $\Sigma_{i} \cdot w_{0}=X_{j(i)}$. For each $j$, there are $\left(\Sigma_{0} \cdot D\left(w_{0}\right): \Sigma_{0}\right)=n / m$ sets $\Sigma_{i}$ such that $\Sigma_{i} \cdot w_{0}=X_{j}$. 
We next compute the terms in the diagram

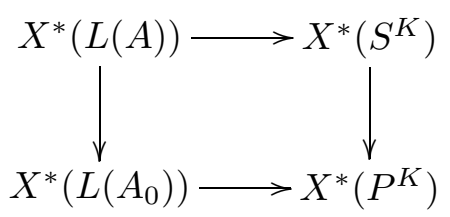

Recall that we have already shown that $X^{*}(L(A)) \rightarrow X^{*}\left(S^{K}\right)$ sends the element $\left[\sigma_{i}\right]$ of $X^{*}(L(A))$ to $\psi_{i}$.

We use the map $\pi \mapsto f_{\pi}^{K}$ (see A.6, A.7) to identify $X^{*}\left(P^{K}\right)$ with

$$
\left\{f \in \mathbb{Z}^{X} \mid \text { there exists an } m \in \mathbb{Z} \text { such that } f+\iota f=m n_{0}\right\} .
$$

Here $n_{0}=\left[K_{w_{0}}: \mathbb{Q}_{p}\right]=\left|D\left(w_{0}\right)\right|$. The map $X^{*}\left(S^{K}\right) \rightarrow X^{*}\left(P^{K}\right)$ is

$$
f=\sum_{\tau \in \Sigma_{K}} f(\tau) \tau \mapsto \sum_{\tau \in \Sigma_{K}} f(\tau) \tau w_{0}=\sum_{w \in X}\left(\sum_{\tau, \tau w_{0}=w} f(\tau)\right) w
$$

(see A.8). When $f=\psi_{i}, w \in X_{j}$ occurs in the right-hand side with nonzero coefficient if and only if $j=j(i)$, in which case its coefficient is $\left|\Sigma_{0} \cap D\left(w_{0}\right)\right|=$ $n_{0} m / n$. Thus the map sends $\psi_{i}$ to $f_{j(i)}$ where $f_{j}$ is the function determined by the conditions

$$
\begin{gathered}
f_{j}(w)= \begin{cases}\left|\Sigma_{0} \cap D\left(w_{0}\right)\right|, & w \in X_{j}, \\
0, & w \in X_{j^{\prime}}, j^{\prime} \neq j,\end{cases} \\
f_{j}(w)+f_{j}(\iota w)=n_{0} \quad \text { for all } w .
\end{gathered}
$$

We identify $X^{*}\left(L\left(A_{0}\right)\right)$ with

$$
\frac{\mathbb{Z}^{\Pi_{A_{0}}}}{\left\{g \mid g=\iota g, \sum g(\pi)=0\right\}}
$$

where $\Pi_{A_{0}}$ is the set of conjugates of $\pi_{A_{0}}$ in $K$ (see A.7). Let $u=\varrho_{0}^{-1} w_{0}$, and let $v_{0}=\sigma_{0}^{-1} w_{0}$. Note that $\sigma_{i}^{-1} w_{0}$ lies over $u_{0}$ and $\left(\iota \sigma_{i}\right)^{-1} w_{0}$ lies over $\iota u_{0}$, $0 \leq i \leq n-1$. Using this, we find that the slope function of the Frobenius germ $\pi_{A_{0}}$ of $A_{0}$ satisfies

$$
s_{\pi_{A_{0}}}(v)= \begin{cases}1 /\left|\Sigma_{E}\left(v_{0}\right)\right|, & v=v_{0}, \\ 0, & v \text { lies over } u_{0}, v \neq v_{0},\end{cases}
$$

where $\Sigma_{E}\left(v_{0}\right)=\left\{\sigma \in \Sigma_{E} \mid \sigma^{-1} w_{0}=v_{0}\right\}$ (see A.8). As $s+\iota s=1$, this determines $s$. Note that

$$
\left|\Sigma_{E}\left(v_{0}\right)\right|=\left(\Sigma_{0} \cdot D\left(w_{0}\right): \Sigma_{0}\right)=\left(D\left(w_{0}\right): \Sigma_{0} \cap D\left(w_{0}\right)\right) .
$$

Note also that $X_{0}$ is the set of $w \in X$ lying over the prime $\sigma_{0} v_{0}$ in $\sigma_{0} E$. For 
any $\tau \in \Sigma_{i}$ (i.e., such that $\tau \circ \sigma_{0}=\sigma_{i}$ ), the diagram

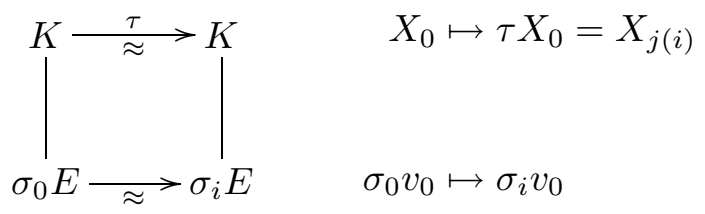

shows that $X_{j(i)}$ is the set of $w \in X$ lying over $\sigma_{i} v_{0}$ in $\sigma_{i} E_{0}$. In other words, $X_{j(i)}$ is the set of $w \in X$ such that $\sigma_{i}^{-1} w=v_{0}$. For $\sigma \in \Sigma_{E}, \sigma \pi_{A_{0}}$ is the Weil germ in $K$ with

$$
f_{\sigma \pi_{A_{0}}}^{K}(w)=s_{\sigma \pi_{A_{0}}}(w) \cdot n_{0}=s_{\pi_{A_{0}}}\left(\sigma^{-1} w\right) \cdot n_{0} .
$$

When $\sigma=\sigma_{i}$ and $w \in X_{j}$, this becomes

$$
f_{\sigma_{i} \pi_{A_{0}}}^{K}(w)= \begin{cases}\left|\Sigma_{0} \cap D\left(w_{0}\right)\right|, & j=j(i), \\ 0, & j \neq j(i) .\end{cases}
$$

Thus, $f_{\sigma_{i} \pi_{A_{0}}}=f_{j(i)}$. In particular, $\sigma_{i} \pi_{A_{0}}$ depends only on $j(i)$. As the functions $f_{j}$ are distinct, we see that

$$
\Pi_{A_{0}}=\left\{\pi_{0}, \ldots, \pi_{m-1}, \iota \pi_{0}, \ldots, \iota \pi_{m-1}\right\}
$$

where $\pi_{j(i)}=\sigma_{i} \pi_{A_{0}}$. The map $X^{*}(L(A)) \rightarrow X^{*}\left(L\left(A_{0}\right)\right)$ sends $\left[\sigma_{i}\right]$ to $\left[\pi_{j(i)}\right]$, and the map $X^{*}\left(L\left(A_{0}\right)\right) \rightarrow X^{*}\left(P^{K}\right)$ sends $\left[\pi_{j}\right]$ to $f_{j}$.

We have now computed all the terms in the diagram $(* * \mathrm{~A})$. It is clear that it commutes.

We next compute the terms in the diagram

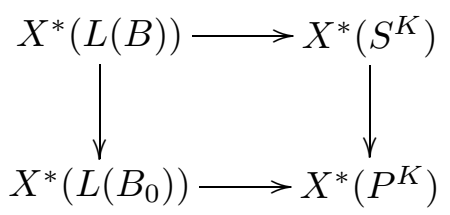

Recall that $X^{*}(L(B))$ has basis $\left[\varrho_{0}\right],\left[\iota \varrho_{0}\right]$, and that the map $X^{*}(L(B)) \rightarrow$ $X^{*}\left(S^{K}\right)$ sends $\left[\varrho_{0}\right]$ to $\psi$ and $\left[\iota \varrho_{0}\right]$ to $\iota \psi$. Here $\psi$ is the characteristic function of $\bigcup \Sigma_{i}$. Clearly, $\mathbb{Q}\left[\pi_{B_{0}}\right]=Q$, and $X^{*}\left(L\left(B_{0}\right)\right)=\mathbb{Z}^{\Sigma_{Q}}$. The left-hand vertical map in the diagram is therefore the identity map. Let $f \in \mathbb{Z}^{X}$ be the function

$$
f(w)= \begin{cases}n_{0} & \text { if } \varrho_{0}^{-1} w=u_{0} \\ 0 & \text { otherwise }\end{cases}
$$

Then $f \in X\left(P^{K}\right)$ and the bottom map sends $\left[\varrho_{0}\right] \mapsto f$. The right-hand vertical map sends $\psi$ to $f$.

On combining the diagrams $(* * \mathrm{~A})$ and $(* * \mathrm{~B})$, we get the right-hand square in $(* *)$. It remains to compute the kernel of $X^{*}\left(L\left(A_{0} \times B_{0}\right)\right) \rightarrow$ 
$X^{*}\left(P^{K}\right)$. Note that

$$
X^{*}\left(L\left(A_{0} \times B_{0}\right)\right)=\frac{\mathbb{Z}^{\Sigma_{\Pi_{A_{0}}} \sqcup \Sigma_{Q}}}{\left\{g \mid g=\iota g \text { and } \sum g(y)=0\right\}} .
$$

The elements $\left[\pi_{0}\right], \ldots,\left[\pi_{m-1}\right],\left[\varrho_{0}\right],\left[\varrho_{0}+\iota \varrho_{0}\right]$ form a basis for $X^{*}\left(L\left(A_{0} \times B_{0}\right)\right)$. They are mapped respectively to $f_{0}, \ldots, f_{m-1}, f, f+\iota f$ in $X^{*}\left(P^{K}\right)$. Clearly,

$$
\frac{n}{m}\left(f_{0}+\ldots+f_{m-1}\right)+(n-2) f=(n-1)(f+\iota f),
$$

and any relation among $f_{0}, \ldots, f_{m-1}, f, f+\iota f$ is a multiple of this one. Therefore, the kernel of $X^{*}\left(L\left(A_{0} \times B_{0}\right)\right) \rightarrow X^{*}\left(P^{K}\right)$ is the free $\mathbb{Z}$-module of rank one generated by

$$
\chi_{0}=\left[\frac{n}{m}\left(\pi_{0}+\ldots+\pi_{m-1}\right)+(n-2) \varrho_{0}-(n-1)\left(\varrho_{0}+\iota \varrho_{0}\right)\right] .
$$

The map $X^{*}(L(A \times B)) \rightarrow X^{*}\left(L\left(A_{0} \times B_{0}\right)\right)$ sends $\chi$ to $\chi_{0}$, and so we have obtained the diagram $(* *)$.

We now prove Theorem 1.5. The group $L\left(A_{0} \times B_{0}\right)$ acts on the space $W\left(A_{0}, B_{0}\right)$ through the characters $\chi_{0}$ and $\iota \chi_{0}=-\chi_{0}$. Because $\chi_{0}$ is trivial on $P\left(A_{0} \times B_{0}\right), W\left(A_{0} \times B_{0}\right)$ consists of Tate classes, and because $\chi_{0}$ is nontrivial on $L\left(A_{0} \times B_{0}\right)$, the classes are exotic. The group $L\left(A_{0} \times B_{0}\right)$ acts on no other subspace of a space $H^{2 r}\left(A_{0} \times B_{0}^{n-2}, \mathbb{Q}_{\ell}(r)\right)$ through the character $\chi_{0}$, and so $W\left(A_{0} \times B_{0}\right)$ contains all the exotic Tate classes on $A_{0} \times B_{0}^{n-2}$.

From $(* *)$, we obtain an exact commutative diagram

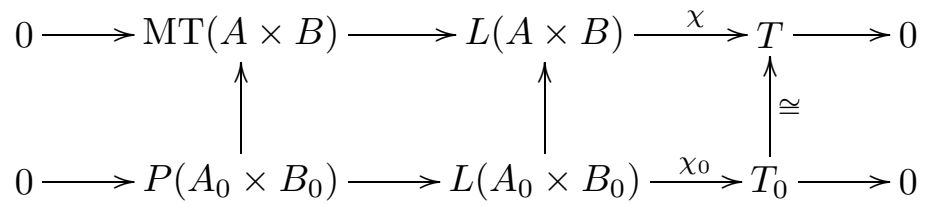

It follows that

$$
P\left(A_{0} \times B_{0}\right)=L\left(A_{0} \times B_{0}\right) \cap \mathrm{MT}(A \times B) .
$$

If some exotic Hodge class on $A \times B^{n-2}$ is algebraic, then the Hodge conjecture holds for all powers of $A \times B^{n-2}$ (see Theorem 1.4), and so Theorem 1.5(b) follows from Corollary 1.2.

REMARK 2.1. It follows from the above calculations that $P\left(A_{0}\right)=L\left(A_{0}\right)$ and $P\left(B_{0}\right)=L\left(B_{0}\right)$, and so all Tate classes on $A_{0}$ and $B_{0}$ are Lefschetz.

Remark 2.2. Choose $E$ to be Galois over $\mathbb{Q}$, and identify it with $K$. In this case, the maps $X^{*}(L(A \times B)) \rightarrow X^{*}\left(S^{K}\right)$ and $X^{*}\left(L\left(A_{0} \times B_{0}\right)\right) \rightarrow$ 
$X^{*}\left(P^{K}\right)$ are surjective, and so we obtain an exact commutative diagram

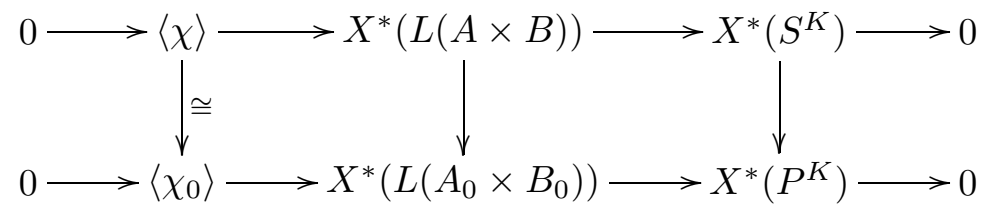

The vertical arrows are surjective, and so

$$
0 \rightarrow \operatorname{Ker}\left(X^{*}(L(A \times B)) \rightarrow X^{*}\left(L\left(A_{0} \times B_{0}\right)\right)\right) \rightarrow X^{*}\left(S^{K}\right) \rightarrow X^{*}\left(P^{K}\right) \rightarrow 0
$$

is exact. Hence

$$
0 \rightarrow P^{K} \rightarrow S^{K} \rightarrow L(A \times B) / L\left(A_{0} \times B_{0}\right)
$$

is exact, which implies that

$$
0 \rightarrow P^{K} \rightarrow S^{K} \rightarrow L^{K} / T^{K}
$$

is exact (notations as in Milne 1999b) because $L(A \times B) / L\left(A_{0} \times B_{0}\right)$ $\rightarrow L^{K} / T^{K}$ is injective. Therefore

$$
P^{K}=S^{K} \cap T^{K} \quad \text { (intersection inside } L^{K} \text { ), }
$$

and we recover ibid., Theorem 6.1.

\section{Appendix A. Abelian varieties with many endomorphisms}

A.1. Notations. Throughout, $\mathbb{Q}^{\text {al }}$ is the algebraic closure of $\mathbb{Q}$ in $\mathbb{C}$, and $\Gamma=\operatorname{Gal}\left(\mathbb{Q}^{\text {al }} / \mathbb{Q}\right)$. Complex conjugation on $\mathbb{C}$, or a subfield of $\mathbb{C}$, is denoted by $\iota$ or $x \mapsto \bar{x}$. In A.8, we fix a prime $w_{0}$ of $\mathbb{Q}^{\text {al }}$ dividing $p$, and denote the residue field at $w_{0}$ by $\mathbb{F}$. We denote the restriction of $w_{0}$ to a subfield of $\mathbb{Q}^{\text {al }}$ by the same symbol. For a finite étale $\mathbb{Q}$-algebra $E, \Sigma_{E}=\operatorname{Hom}\left(E, \mathbb{Q}^{\text {al }}\right)$. For a subfield $K$ of $\mathbb{Q}^{\text {al }}$ Galois over $\mathbb{Q}, \Sigma_{K}$ can be identified with $\operatorname{Gal}(K / \mathbb{Q})$.

A $C M$-algebra $E$ is a finite product of finite field extensions of $\mathbb{Q}$ admitting an involution $\iota_{E}$ that is nontrivial on each factor and such that $\sigma\left(\iota_{E} x\right)=\overline{\sigma(x)}$ for all $\sigma: E \rightarrow \mathbb{C}$; equivalently, $E$ is a finite product of CM-fields.

For a finite set $Y, \mathbb{Z}^{Y}$ denotes the set of functions $f: Y \rightarrow \mathbb{Z}$. We sometimes denote such a function by $\sum f(y) y$; for example, the function $f=y_{1}$ takes the value 1 on $y_{1}$ and 0 on all $y \neq y_{1}$.

For a group of multiplicative type $T$ over $\mathbb{Q}, X^{*}(T)={ }_{\mathrm{df}} \operatorname{Hom}\left(T_{\mathbb{Q}^{\mathrm{al}}}, \mathbb{G}_{m}\right)$ is the character group. We often use the pairing

$$
\chi, \mu \mapsto\langle\chi, \mu\rangle \stackrel{\text { df }}{=} \chi \circ \mu: X^{*}(T) \times X_{*}(T) \rightarrow \operatorname{End}\left(\mathbb{G}_{m}\right) \cong \mathbb{Z}
$$

to identify the cocharacter group $X_{*}(T)=_{\text {df }} \operatorname{Hom}_{\mathbb{Q}^{a l}}\left(\mathbb{G}_{m}, T_{\mathbb{Q}^{a l}}\right)$ of $T$ with the $\mathbb{Z}$-linear dual of $X^{*}(T)$. The group $\Gamma$ acts on $X_{*}(T)$ and $X^{*}(T)$, and $\langle\gamma \chi, \gamma \mu\rangle=\langle\chi, \mu\rangle$ for all $\gamma \in \Gamma, \chi \in X^{*}(T)$, and $\mu \in X_{*}(T)$. 
Let $\varrho: T \rightarrow \mathrm{GL}(V)$ be a representation of a group $T$ of multiplicative type on a finite-dimensional $\mathbb{Q}$-vector space $V$. For any subfield $\Omega$ of $\mathbb{C}$ that splits $T$, there is a decomposition

$$
V \otimes_{\mathbb{Q}} \Omega \cong \bigoplus_{\chi \in X^{*}(T)} V_{\chi}
$$

where $V_{\chi}$ is the subspace of $V \otimes_{\mathbb{Q}} \Omega$ on which $T$ acts through $\chi$. If $V_{\chi}$ is nonzero, then we say that $\chi$ occurs in $V$. When $\Omega$ is Galois over $\mathbb{Q}$, a subspace $\bigoplus_{\chi \in \Xi} V_{\chi}, \Xi \subset X^{*}(T)$, is defined over $\mathbb{Q}$ (i.e., of the form $W \otimes_{\mathbb{Q}} \Omega$ for some subspace $W \subset V$ ) if and only if $\Xi$ is stable under $\Gamma$. The subspace of vectors in $V$ fixed by $T$ (in the sense of Milne 1999a, §3) is denoted $V^{T}$.

For a finite étale $\mathbb{Q}$-algebra $E,\left(\mathbb{G}_{m}\right)_{E / \mathbb{Q}}={ }_{\mathrm{df}} \operatorname{Res}_{E / \mathbb{Q}}\left(\mathbb{G}_{m}\right)$ (Weil restriction of scalars), so that $X^{*}\left(\left(\mathbb{G}_{m}\right)_{E / \mathbb{Q}}\right)=\mathbb{Z}^{\Sigma_{E}}$. Under this identification, an element $f=\sum f(\sigma) \sigma$ of $\mathbb{Z}^{\Sigma_{E}}$ maps an element $a$ of $E^{\times}=\left(\mathbb{G}_{m}\right)_{E / \mathbb{Q}}(\mathbb{Q})$ to $a^{f}=\prod(\sigma a)^{f(\sigma)}$. We sometimes identify a subset $\Delta$ of $\Sigma_{E}$ with the character $\sum_{\sigma \in \Delta} \sigma$; for example, if $V$ is an $E$-vector space, then $\left(V^{\otimes r} \otimes \Omega\right)_{\Delta}$ is the subspace on which $a \in E$ acts as $\prod_{\sigma \in \Delta} \sigma a$.

There is a natural correspondence $\left({ }^{3}\right)$ between

- triples $(T, w, t)$ comprising a group of multiplicative type $T$, a cocharacter $w$ of $T$, and a character $t$, all defined over $\mathbb{Q}$, such that $t \circ w=-2$; and

- pairs $\left(T_{0}, \varepsilon\right)$ comprising a group of multiplicative type $T_{0}$ and an element $\varepsilon$ of order 1 or 2 in $T_{0}(\mathbb{Q})$.

Given $(T, w, t)$, define $T_{0}$ to be the kernel of $t$ and $\varepsilon$ to be $w(-1)$. Conversely, given $\left(T_{0}, \varepsilon\right)$, define $T$ by the diagram

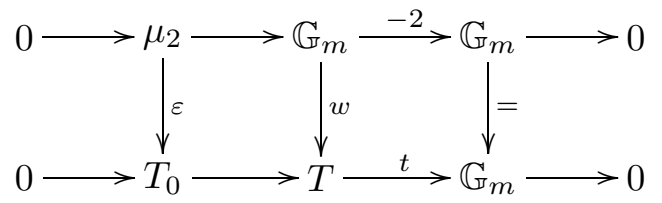

in which $T=\left(T_{0} \times \mathbb{G}_{m}\right) / \mu_{2}$. If $\left(T_{1}, w_{1}, t_{1}\right) \subset\left(T_{2}, w_{2}, t_{2}\right)$, then $T_{1}=T_{2}$ if and only if $\left(T_{1}\right)_{0}=\left(T_{2}\right)_{0}$.

Let $\varrho_{0}: T_{0} \rightarrow \mathrm{GL}(V)$ be a representation of $T_{0}$ such that $\varrho_{0}(\varepsilon)$ acts on $V$ as multiplication by the scalar -1 , and let $W$ be a one-dimensional vector space with basis $e$. Then

$$
(x, y) \mapsto\left(\varrho_{0}(x) \cdot y, y^{-2}\right): T_{0} \times \mathbb{G}_{m} \rightarrow \mathrm{GL}(V) \times \mathrm{GL}(W)
$$

sends $(\varepsilon, \varepsilon)$ to 1 , and therefore defines a homomorphism $\varrho: T \rightarrow \operatorname{GL}(V) \times$ $\mathrm{GL}(W)$. Note that $(\varrho \circ w)(y)$ acts on $V$ as $y$, and that the composite of $\varrho$

$\left({ }^{3}\right)$ Experts will recognize the Tannakian significance of this correspondence (Saavedra 1972, V 3.1.4; Deligne and Milne 1982, p. 190). 
with the projection to $\operatorname{GL}(W)$ is $t$. Let $s \in V^{\otimes i}$. If $s$ is fixed by $T_{0}$, then $i$ is even. There is a one-to-one correspondence

$$
s \leftrightarrow s \otimes e^{\otimes j}
$$

between the elements $s$ of $V^{\otimes 2 j}$ fixed by $T_{0}$ and the elements of $V^{\otimes 2 j} \otimes W^{\otimes j}$ fixed by $T$.

For a smooth projective variety $X, \mathcal{Z}^{r}(X)$ is the space of algebraic cycles on $X$ of codimension $r$ with coefficients in $\mathbb{Q}$, and $\mathcal{Z}_{\text {num }}^{r}(X)$ is the quotient of $\mathcal{Z}^{r}(X)$ by numerical equivalence. The space $\mathcal{Z}_{\text {num }}^{*}(X)=\bigoplus_{r} \mathcal{Z}_{\text {num }}^{r}(X)$ becomes a $\mathbb{Q}$-algebra under intersection product. An algebraic class in a cohomology group with coefficients in a field $\Omega$ is an element of the $\Omega$ subspace spanned by the classes of algebraic cycles.

For an abelian variety $A$ over an algebraically closed field $k$ of characteristic zero, we often implicitly assume that there is given an embedding $\sigma: k \rightarrow \mathbb{C}$ so that we can define $H^{r}(A, \mathbb{Q})$ to be $r$ th cohomology group of the complex manifold $(\sigma A)(\mathbb{C})$. We let $\operatorname{Hom}^{0}(A, B)=\operatorname{Hom}(A, B) \otimes_{\mathbb{Z}} \mathbb{Q}$.

For Hodge structures and class field theory, we follow the usual conventions of those areas rather than the conventions of Deligne used in my previous papers. For example, $z \in \mathbb{C}^{\times}$acts on a Hodge structure of type $(r, s)$ as $z^{r} \bar{z}^{s}$, and the Artin reciprocity maps send prime elements to the Frobenius element $x \mapsto x^{q}$.

We sometimes use $[x]$ to denote an equivalence class containing $x$, and $|X|$ to denote the order of a finite set $X$.

For an explanation of the various cohomology groups of varieties, and their Tate twists, see Deligne 1982, $\S 1$.

This section summarizes results due to many mathematicians. Omitted proofs can be found in Milne 1999a, 1999b, Tate 1968/69, or in the references for those articles.

A.2. Generalities. Let $A$ be an abelian variety over an algebraically closed field $k$. The reduced degree $\left(^{4}\right)$ of the $\mathbb{Q}$-algebra $\operatorname{End}^{0}(A)$ is $\leq 2 \operatorname{dim} A$, and when equality holds the abelian variety is said $\left(^{5}\right)$ to have many endomorphisms. An isotypic $\left({ }^{6}\right)$ abelian variety has many endomorphisms if and only if $\operatorname{End}^{0}(A)$ contains a field of degree $2 \operatorname{dim} A$ over $\mathbb{Q}$, and an arbitrary

$\left({ }^{4}\right)$ Let $R$ be a semisimple algebra of finite degree over $\mathbb{Q}$. Then $R$ is a product of simple algebras, say, $R=R_{1} \times \ldots \times R_{m}$, and the centre $E_{i}$ of each $R_{i}$ is a field. The reduced degree $[R: \mathbb{Q}]_{\text {red }}$ of $R$ over $\mathbb{Q}$ is defined to be $\sum_{i=1}^{m}\left[R_{i}: E_{i}\right]^{1 / 2}\left[E_{i}: \mathbb{Q}\right]$.

$\left({ }^{5}\right)$ Often such an abelian variety is said to admit "complex multiplication", but this conflicts with classical terminology - see Lange and Birkenhake 1992, p. 268. Also "multiplication" for "endomorphism" seems archaic.

$\left({ }^{6}\right)$ An abelian variety is said to be isotypic if it is isogenous to a power of a simple abelian variety. 
abelian variety has many endomorphisms if and only if each isotypic isogeny factor of it does. Equivalent conditions:

(a) the $\mathbb{Q}$-algebra $\operatorname{End}^{0}(A)$ contains an étale subalgebra of degree $2 \operatorname{dim} A$ over $\mathbb{Q}$;

(b) for a Weil cohomology $X \mapsto H^{*}(X)$ with coefficient field $\Omega$, the centralizer of $\operatorname{End}^{0}(A)$ in $\operatorname{End}_{\Omega}\left(H^{1}(A)\right)$ is commutative (in which case it equals $C(A) \otimes_{\mathbb{Q}} \Omega$ where $C(A)$ is the centre of $\left.\operatorname{End}^{0}(A)\right)$;

(c) (characteristic zero) $A$ has CM-type, i.e., its Mumford-Tate group (see A.3 below) is commutative (hence a torus);

(d) (characteristic $p \neq 0$ ) $A$ is isogenous to an abelian variety defined over $\mathbb{F}$ (theorems of Tate and Grothendieck).

Let $k \subset k^{\prime}$ be algebraically closed fields. The functor $A \mapsto A_{k^{\prime}}$ from the category of abelian varieties over $k$ to the similar category over $k^{\prime}$ is fully faithful, because the map on torsion points $A(k)_{\text {tors }} \rightarrow A\left(k^{\prime}\right)_{\text {tors }}$ is bijective and $A(k)_{\text {tors }}$ is Zariski dense in $A$. That the functor becomes essentially surjective on the categories of abelian varieties with many endomorphisms up to isogeny is a result of Grothendieck (Oort 1973). Thus, in large part, the theory of abelian varieties with many endomorphisms up to isogeny over an algebraically closed field depends only on the characteristic of the field.

\section{A.3. The groups attached to an abelian variety with many endo-} morphisms. Let $A$ be an abelian variety with many endomorphisms over an algebraically closed field $k$, and let $C(A)$ be the centre of $\operatorname{End}^{0}(A)$. Every Rosati involution on $\operatorname{End}^{0}(A)$ stabilizes $C(A)$, and the different Rosati involutions restrict to the same involution $\left({ }^{7}\right)$ on $C(A)$, which we denote ${ }^{\dagger}$. Each factor of $C(A)$ is either a CM-field, on which ${ }^{\dagger}$ acts as complex conjugation, or is $\mathbb{Q}$.

The Lefschetz group. We define $L(A)_{0}$ to be the group of multiplicative type over $\mathbb{Q}$ such that, for all commutative $\mathbb{Q}$-algebras $R$,

$$
L(A)_{0}(R)=\left\{\alpha \in C(A) \otimes R \mid \alpha \alpha^{\dagger}=1\right\} .
$$

Let $\varepsilon=-1 \in L(A)_{0}(\mathbb{Q})$, and let $(L(A), w, t)$ be the triple associated (as in A.1) with $\left(L(A)_{0}, \varepsilon\right)$.

Then

$$
L(A)(\mathbb{Q}) \cong\left\{\alpha \in C(A)^{\times} \mid \alpha \alpha^{\dagger} \in \mathbb{Q}^{\times}\right\},
$$

and, on $\mathbb{Q}$-points, $w$ is $x \mapsto x$ and $t$ is $x \mapsto\left(x x^{\dagger}\right)^{-1}$.

The motivic group. Because $L(A)_{0}$ is a subgroup of $\operatorname{End}^{0}(A)^{\times}$, it acts on $\mathcal{Z}_{\text {num }}^{*}\left(A^{s}\right)$ for all $s$, and we define $M(A)_{0}$ to be the largest algebraic subgroup

$\left({ }^{7}\right)$ The Rosati involution defined by a polarization $\lambda: A \rightarrow A^{\vee}$ is $\alpha \mapsto \alpha^{\dagger}=$ $\lambda^{-1} \circ \alpha^{\vee} \circ \lambda$. Let $\mu$ be a second polarization, and let $\beta=\lambda^{-1} \circ \mu$. If $\alpha \in C(A)$, then $\alpha^{\dagger} \in C(A)$ and $\mu^{-1} \circ \alpha^{\vee} \circ \mu=\beta^{-1} \circ \alpha^{\dagger} \circ \beta=\alpha^{\dagger}$. 
of $L(A)_{0}$ acting trivially on these $\mathbb{Q}$-algebras. Then $-1 \in M(A)_{0}(\mathbb{Q})$, and we let $(M(A), w, t)$ be the triple associated with $\left(M(A)_{0},-1\right)$.

The Mumford-Tate group. When $k$ has characteristic zero, $L(A)_{0}$ acts on the $\mathbb{Q}$-algebra of Hodge classes on $A^{s}$ for all $s$, and we define $\operatorname{MT}(A)_{0}$ to be the subgroup of $L(A)_{0}$ fixing the elements of these $\mathbb{Q}$-algebras. Again $-1 \in \operatorname{MT}(A)_{0}$, and we let $(\operatorname{MT}(A), w, t)$ be the triple associated with $\left(\mathrm{MT}(A)_{0},-1\right)$.

The group $P$. Let $k=\mathbb{F}$. A model $A_{1}$ of $A$ over a finite field $\mathbb{F}_{q}$ defines a Weil $q$-number $\pi_{1}$, whose class $\pi_{A}$ in $W\left(p^{\infty}\right)$ (see A.6 below) is independent of the choice of $A_{1}$. The group $P(A)$ is defined to be the smallest algebraic subgroup of $L(A)$ containing some power of $\pi_{1}$ - again, it is independent of the choice of $A_{1}$.

Let $\pi_{1}$ be a Weil $p^{2 n}$-number representing $\pi_{A}$. Then $\pi_{1} / p^{n} \in L(A)_{0}$, and $P(A)_{0}$ is the smallest algebraic subgroup of $L(A)_{0}$ containing some power of $\pi_{1} / p^{n}$.

Let $H^{*}$ be a Weil cohomology with coefficients in a field $\Omega$. Since $L(A)_{0} \subset$ $\left(\mathbb{G}_{m}\right)_{C(A) / \mathbb{Q}}$, there is a natural action of $L(A)_{0}$ on $H^{1}(A, \Omega)$, and $\varepsilon$ acts as -1 . Hence (see A.1) there is a natural action of $L(A)$ on

$$
H^{r}\left(A^{s}, \Omega\right)(m) \cong\left(\bigwedge^{r}\left(\bigoplus_{s \text { copies }} H^{1}(A, \Omega)\right)\right) \otimes(\Omega(1))^{\otimes m} .
$$

LEMmA. Let $A$ be an abelian variety with many endomorphisms over an algebraically closed field $k$, and let $H^{*}$ be a Weil cohomology with coefficients in a field $\Omega$. Let $H^{2 *}\left(A^{s}\right)(*)=\bigoplus_{r} H^{2 r}\left(A^{s}\right)(r)$. Then, for all $s$,

(a) $H^{2 *}\left(A^{s}\right)(*)^{L(A)}$ is the $\Omega$-subalgebra of $H^{2 *}\left(A^{s}\right)(*)$ generated by the classes of divisors on $A^{s}$ (i.e., it is the space of Lefschetz classes);

(b) $H^{2 *}\left(A^{s}\right)(*)^{M(A)}$ is the space of algebraic classes in $H^{2 *}\left(A^{s}\right)(*)$, provided numerical equivalence coincides with homological equivalence for $H^{*}$;

(c) $H^{2 *}\left(A^{s}\right)(*)^{\mathrm{MT}(A)}$ is the space of Hodge classes on $A^{s}$ when $k$ has characteristic zero and $H^{*}$ is the cohomology defined by an embedding $k \rightarrow \mathbb{C}$;

(d) $H^{2 *}\left(A^{s}, \mathbb{Q}_{\ell}(*)\right)^{P(A)}$ is the space of $\ell$-adic Tate classes on $A^{s}$ when $k=\mathbb{F}$ and $H^{*}$ is $\ell$-adic étale cohomology.

Proof. Statement (a) is proved in Milne 1999a (Theorem 4.4).

For (b), recall that theorems of Jannsen and Deligne show that the category of motives over $k$, based on abelian varieties and defined using the numerical equivalence classes of algebraic cycles as correspondences, is Tannakian (Jannsen 1992). Almost by definition, $M(A)$ is the fundamental group of the Tannakian subcategory $\langle A\rangle^{\otimes}$ of this category generated by $A$ and the Tate object. When numerical equivalence coincides with homological equivalence, the Weil cohomology defines a fibre functor, and there is 
a natural map

$$
\operatorname{Hom}\left(\mathbb{1}, h^{2 r}\left(A^{s}\right)(r)\right) \otimes_{\mathbb{Q}} \Omega \rightarrow \operatorname{Hom}_{\Omega}\left(\Omega, H^{2 r}\left(A^{s}\right)(r)\right)^{M(A)},
$$

which the theory of Tannakian categories shows to be bijective. But

$$
\operatorname{Hom}\left(\mathbb{1}, h^{2 r}\left(A^{s}\right)(r)\right)=\mathcal{Z}_{\text {num }}^{r}\left(A^{s}\right) .
$$

Statement (c) is proved in Deligne 1982 (see the proof of 3.4).

Almost by definition of $P(A), H^{2 *}\left(A^{s}, \mathbb{Q}_{\ell}(*)\right)^{P(A)}$ consists of the classes fixed by the Frobenius germ $\pi_{A}$, and these are exactly the Tate classes.

Thus (Deligne 1982, 3.1), under the hypotheses in each part of the lemma, knowing the group ? $(A)_{\Omega}$ is equivalent to knowing the corresponding spaces of fixed classes: ? $(A)_{\Omega}$ is the largest algebraic subgroup of $\operatorname{GL}\left(H^{1}(A)\right)$ $\times \mathbb{G}_{m}$ fixing the particular classes on all $A^{s}$, and the particular classes are exactly those fixed by $?(A)_{\Omega}$.

TheOREM. (a) For any abelian variety $A$ with many endomorphisms over an algebraically closed field $k$ of characteristic zero, $\operatorname{MT}(A) \subset M(A) \subset$ $L(A)$, and

(i) the Hodge conjecture holds for all powers of $A$ if and only if $\operatorname{MT}(A)=M(A)$

(ii) all Hodge classes on all powers of $A$ are Lefschetz if and only if $\operatorname{MT}(A)=L(A)$.

When $k=\mathbb{Q}^{\text {al }}$, "Hodge" can be replaced by "Tate" in the above statements.

(b) For any abelian variety $A_{0}$ over $\mathbb{F}, P\left(A_{0}\right) \subset M\left(A_{0}\right) \subset L\left(A_{0}\right)$, and

(i) all $\ell$-adic Tate classes on all powers of $A_{0}$ are algebraic for one (or all) $\ell$ if and only if $P\left(A_{0}\right)=M\left(A_{0}\right)$;

(ii) all $\ell$-adic Tate classes on all powers of $A_{0}$ are Lefschetz for one (or all) $\ell$ if and only if $P\left(A_{0}\right)=L\left(A_{0}\right)$.

Proof. Since every character of $L(A)$ occurs in a space of the form $H^{r}\left(A^{s}\right)(m)$, we see that the subgroups of $L(A)$ are determined by their invariants in these spaces. Thus (a) of the Theorem is an immediate consequence of the Lemma. That "Hodge" can be replaced by "Tate" follows from Pohlmann 1968.

If $P\left(A_{0}\right)=M\left(A_{0}\right)$, then the Lemma shows that the $\ell$-adic Tate conjecture holds for all powers of $A_{0}$ and all $\ell$ in the set in Proposition B.2, but if the $\ell$-adic Tate conjecture holds for one $\ell$ then it holds for all (Tate $1994,2.9)$. Conversely, if the $\ell$-adic Tate conjecture holds for all powers of $A_{0}$ and a single $\ell$, then numerical equivalence coincides with $\ell$-homological equivalence for that $\ell$ (Tate $1994,2.9,(\mathrm{c}) \Rightarrow(\mathrm{b})$ ), and the preceding Lemma then shows that $P\left(A_{0}\right)_{\mathbb{Q}_{\ell}}=M\left(A_{0}\right)_{\mathbb{Q}_{\ell}}$. As $P\left(A_{0}\right) \subset M\left(A_{0}\right)$, this implies that $P\left(A_{0}\right)=M\left(A_{0}\right)$. 
The proof of the remaining statement is similar.

ExAmple. If $A$ has dimension 1 , then either $\operatorname{End}^{0}(A)$ is a quadratic imaginary field $E$ or a quaternion algebra $D$ with centre $\mathbb{Q}$. In the first case, all the groups attached to $A$ equal $\left(\mathbb{G}_{m}\right)_{E / \mathbb{Q}}$ and in the second, all the groups attached to $A$ equal $\mathbb{G}_{m}$. Hence, there are no exotic Hodge or Tate classes on any power of an elliptic curve, and the Hodge and Tate conjectures hold.

REMARK. Let $A$ be an abelian variety with many endomorphisms over $\mathbb{Q}^{\text {al }}$, and let $A_{0}$ be its reduction to an abelian variety over $A_{0}$. The reduction map $\operatorname{End}^{0}(A) \rightarrow \operatorname{End}^{0}\left(A_{0}\right)$ is injective, and the image of the centre of $\operatorname{End}^{0}(A)$ contains the centre of $\operatorname{End}^{0}\left(A_{0}\right)$ (because the latter is generated as a $\mathbb{Q}$-algebra by a Frobenius element which lifts to an element of the centre of $\left.\operatorname{End}^{0}(A)\right)$. Therefore, $L(A) \supset L\left(A_{0}\right)$.

\section{A.4. Classification over $\mathbb{C}$ of abelian varieties with many en-} domorphisms. Let $E$ be a CM-algebra. A CM-type on $E$ is the choice of one out of every pair of complex conjugate homomorphisms $E \rightarrow \mathbb{C}$. It can variously be considered as:

(a) a partition $\Sigma_{E}=\Phi \cup \iota \Phi$;

(b) a function $\varphi: \Sigma_{E} \rightarrow \mathbb{Z}$ such that, for all $\sigma, \varphi(\sigma) \geq 0$ and $\varphi(\sigma)+$ $\varphi(\iota \sigma)=1$

(c) the choice of an isomorphism $E \otimes_{\mathbb{Q}} \mathbb{R} \rightarrow \mathbb{C}^{\Sigma_{F}}$ where $F$ is the product of the maximal real subfields of the factors of $E$.

Here $\Phi$ is the support of $\varphi$ and $\varphi$ is the characteristic function of $\Phi$.

Let $A$ be a simple abelian variety over $\mathbb{C}$ with many endomorphisms. Then $\operatorname{End}^{0}(A)$ is a CM-field $E$, and the action of $E$ on $\Gamma\left(A, \Omega^{1}\right)$ defines a CM-type $\Phi$ on $E$, which is primitive, i.e., not the extension of a CM-type on a proper CM-subfield of $E$. The map $A \mapsto(E, \Phi)$ defines a bijection from the set of isogeny classes of simple abelian varieties over $\mathbb{C}$ with many endomorphisms to the set of isomorphism classes of pairs $(E, \Phi)$. It remains to classify the pairs $(E, \Phi)$.

Fix a (large) CM-field $K \subset \mathbb{Q}^{\text {al }}$, finite and Galois over $\mathbb{Q}$. The Serre group $S^{K}$ of $K$ is the quotient of $\left(\mathbb{G}_{m}\right)_{K / \mathbb{Q}}$ whose character group consists of the $f \in \mathbb{Z}^{\Sigma_{K}}$ for which there is an integer wt $(f)$ (the weight of $f$ ) such that $f(\tau)+f(\iota \tau)=\operatorname{wt}(f)$ for all $\tau \in \Sigma_{K}$, that is,

$$
X^{*}\left(S^{K}\right)=\left\{f \in \mathbb{Z}^{\Sigma_{K}} \mid f+\iota f \text { is constant }\right\} .
$$

The reflex field of $(E, \Phi)$ is the fixed field of the subgroup $\{\tau \in \Gamma \mid$ $\tau \Phi=\Phi\}$ of $\Gamma$. We classify the pairs $(E, \Phi)$ whose reflex field is contained in $K$. Let $\varphi$ be the characteristic function of $\Phi$. For each $\sigma: E \rightarrow \mathbb{Q}^{\text {al }}$ and $\tau \in \operatorname{Gal}\left(\mathbb{Q}^{\text {al }} / \mathbb{Q}\right)$, define

$$
\psi_{\sigma}(\tau)=\varphi\left(\tau^{-1} \circ \sigma\right) .
$$


Then $\psi_{\sigma}(\tau)$ depends only on $\tau \mid K$, and for any $\varrho \in \operatorname{Gal}\left(\mathbb{Q}^{\text {al }} / \mathbb{Q}\right), \psi_{\varrho \circ \sigma}=\varrho \psi_{\sigma}$. Thus, $\left\{\psi_{\sigma}\right\}$ is a $\Gamma$-orbit in $\mathbb{Z}^{\Sigma_{K}}$. The map $(E, \Phi) \mapsto\left\{\psi_{\sigma}\right\}$ is a bijection from the set of isomorphism classes of pairs $(E, \Phi)$ comprising a CM-field and a primitive CM-type whose reflex field is contained in $K$ to the set of $\Gamma$-orbits of elements $f$ of $X^{*}\left(S^{K}\right)$ such that $f(\tau) \geq 0$ for all $\tau$ and $\operatorname{wt}(f)=1$.

A.5. Calculation of the groups over $\mathbb{C}$. Let $A$ be an abelian variety with many endomorphisms over $\mathbb{C}$. Then $A$ is isogenous to a product $A_{1}^{s_{1}} \times$ $\ldots \times A_{t}^{s_{t}}$ with the $A_{i}$ simple and pairwise nonisogenous, and

$$
\begin{aligned}
L(A) & \cong L\left(A_{1} \times \ldots \times A_{t}\right) \quad\left(\text { in fact } L(A)_{0} \cong L\left(A_{1}\right)_{0} \times \ldots \times L\left(A_{t}\right)_{0}\right), \\
M(A) & \cong M\left(A_{1} \times \ldots \times A_{t}\right), \\
\operatorname{MT}(A) & \cong \operatorname{MT}\left(A_{1} \times \ldots \times A_{t}\right) .
\end{aligned}
$$

Thus, in the following, we assume that $A$ is a product of pairwise nonisogenous simple abelian varieties. Then $E={ }_{\mathrm{df}} \operatorname{End}^{0}(A)$ is a CM-algebra. The action of $E$ on $H^{1,0}(A)$ defines a CM-type $\Phi$ on $E$, and the Rosati involution is $\iota_{E}$.

The Lefschetz group. The group $L(A)$ is the subgroup of $\left(\mathbb{G}_{m}\right)_{E / \mathbb{Q}}$ whose character group is

$$
\frac{\mathbb{Z}^{\Sigma_{E}}}{\left\{g \mid g=\iota g \text { and } \sum g(\sigma)=0\right\}} .
$$

The weight map $w: \mathbb{G}_{m} \rightarrow L(A)$ corresponds to the map

$$
[g] \mapsto \mathrm{wt}(g) \stackrel{\mathrm{df}}{=} \sum_{\sigma \in \Sigma_{E}} g(\sigma)
$$

on characters, and the homomorphism $t: L(A) \rightarrow \mathbb{G}_{m}$ giving the action of $L(A)$ on the Tate object $\mathbb{Q}(1)$ sends $1 \in X^{*}\left(\mathbb{G}_{m}\right)$ to the element of $X^{*}(L(A))$ represented by $-\sigma-\iota \sigma$ for any $\sigma \in \Sigma_{E}$.

The group $L(A)_{0}$ is the subgroup of $\left(\mathbb{G}_{m}\right)_{E / \mathbb{Q}}$ whose character group is

$$
\frac{\mathbb{Z}^{\Sigma_{E}}}{\{g \mid g=\iota g\}} .
$$

The map $\mu_{2} \rightarrow L(A)_{0}$ corresponds to the map on characters $[g] \mapsto \sum g(\sigma)$ $\bmod 2$.

When $A$ is simple, the map $\sigma \mapsto \psi_{\sigma}$ is bijective and commutes with the action of $\Gamma$, and so it identifies $L(A)$ with the torus whose character group is

$$
\frac{\mathbb{Z}^{\Psi}}{\left\{g \mid g=\iota g \text { and } \sum g(\psi)=0\right\}}, \quad \Psi=\left\{\psi_{\sigma} \mid \sigma \in \Sigma_{E}\right\} .
$$


The Mumford-Tate group. The Hodge decomposition on $H^{r}\left(A^{s}, \mathbb{Q}\right)(m)$ is defined over $\mathbb{Q}^{\text {al }}$, i.e., there is a decomposition

$$
H^{r}\left(A^{s}, \mathbb{Q}\right)(m) \otimes \mathbb{Q}^{\text {al }} \cong \bigoplus_{i+j=r-2 m} H^{r}\left(A^{s}\right)(m)^{i, j}
$$

that becomes the Hodge decomposition when tensored with $\mathbb{C}$. Since $L(A)_{0}$ $\subset\left(\mathbb{G}_{m}\right)_{E / \mathbb{Q}}$, there is a natural action of $L(A)_{0}$ on $H^{1}(A, \mathbb{Q})$, and $\varepsilon$ acts as -1 . Hence (see A.1) there is a natural action of $L(A)$ on

$$
H^{r}\left(A^{s}, \mathbb{Q}\right)(m) \cong\left(\bigwedge^{r}\left(\bigoplus_{s \text { copies }} H^{1}(A, \mathbb{Q})\right)\right) \otimes(\mathbb{Q}(1))^{\otimes m}
$$

For $\chi=[g] \in X^{*}(L(A)),\left(H^{r}\left(A^{s}\right)(m)\right)_{\chi}$ is of Hodge type

$$
\left(\sum_{\sigma \in \Phi} g(\sigma), \sum_{\sigma \in \iota \Phi} g(\sigma)\right) .
$$

Every character of $L(A)$ occurs in $H^{r}\left(A^{s}, \mathbb{Q}\right)(m)$ for some $r, s, m$, and if $[g]$ occurs in $H^{r}\left(A^{s}\right)(m)$, then $\operatorname{wt}(g)=r-2 m$. A character $\chi$ of $L(A)$ is trivial on $\operatorname{MT}(A)$ if and only if $\bigoplus_{\tau \in \Gamma} H^{2 r}\left(A^{s}\right)(r)_{\tau \chi}$ is purely of type $(0,0)$ for some $r, s$ for which the space is nonzero. Hence, a character $\chi=[g]$ of $L(A)$ is trivial on $\operatorname{MT}(A)$ if and only if

$$
\sum_{\sigma \in \Phi} g(\tau \circ \sigma)=0 \quad \text { for all } \tau \in \Gamma .
$$

The motivic group. Let $\chi \in X^{*}(L(A))$. Then $\chi$ is trivial on $M(A)$ if and only if $H^{2 r}\left(A^{s}\right)(r)_{\chi}$ contains a nonzero algebraic class for some $r$ and $s$, in which case the spaces $H^{2 r}\left(A^{s}\right)(r)_{\chi}$ consist entirely of algebraic classes for all $r$ and $s$ (see (b) of the lemma in A.3).

Second description of $\operatorname{MT}(A)$. There is another description of $\operatorname{MT}(A)$ that is useful. Let $K$ be a CM-subfield of $\mathbb{Q}^{\text {al }}$, finite and Galois over $\mathbb{Q}$, and let $S^{K}$ be its Serre group. Let $\tau_{0} \in \Sigma_{K}$ be the given embedding of $K$ into $\mathbb{Q}^{\text {al }}$. Then $f \mapsto f\left(\tau_{0}\right)$ is a cocharacter $\mu^{K}$ of $S^{K}$ with the property that $\mu^{K}+\iota \mu^{K}$ is fixed by $\Gamma$ and so is defined over $\mathbb{Q}$. The pair $\left(S^{K}, \mu^{K}\right)$ is universal: if $T$ is a second torus over $\mathbb{Q}$ and $\mu \in X_{*}(T)$ is defined over $K$ and $\mu+\iota \mu$ is defined over $\mathbb{Q}$, then there is a unique homomorphism $\varrho_{\mu}: S^{K} \rightarrow T$ such that $\left(\varrho_{\mu}\right)_{\mathbb{Q}^{\text {al }}} \circ \mu^{K}=\mu$. On characters, $\varrho_{\mu}$ sends $\chi \in X^{*}(T)$ to the element $f$ of $X^{*}\left(S^{K}\right)$ with $f(\tau)=\langle\chi, \tau \mu\rangle$ for all $\tau$.

Let $A$ be an abelian variety of CM-type $(E, \Phi)$, and let $\mu_{\Phi}$ be the cocharacter of $L(A)$ sending a character $[g]$ of $L(A)$ to $\sum_{\sigma \in \Phi} g(\sigma)$. If $K$ contains the reflex field of $\Phi$, then $\mu_{\Phi}$ is defined over $K$. Moreover $\mu_{\Phi}+\iota \mu_{\Phi}$ is $[g] \mapsto \operatorname{wt}(g)$, which is defined over $\mathbb{Q}$, and so there is a unique homomorphism $\varrho_{\Phi}: S^{K} \rightarrow L(A)$ such that $\varrho_{\Phi} \circ \mu^{K}=\mu_{\Phi}$. It sends a character $g$ of 
$L(A)$ to the character $f$ of $S^{K}$ such that

$$
f(\tau)=\left\langle[g], \tau \mu_{\Phi}\right\rangle=\left\langle\tau^{-1}[g], \mu_{\Phi}\right\rangle=\sum_{\sigma \in \Phi} g(\tau \circ \sigma) .
$$

The image of this homomorphism is $\operatorname{MT}(A)$. It is obvious that this description agrees with the previous one.

REMARK. The roles of $K$ and $E$ should be carefully distinguished. The first is a "large" CM-subfield of $\mathbb{Q}^{\text {al }}$ Galois over $\mathbb{Q}$; the second is a finite product of abstract CM-fields acting on $A$. The field $K$ contains the reflex field of $(E, \Phi)$. There are homomorphisms

$$
\left(\mathbb{G}_{m}\right)_{K / \mathbb{Q}} \rightarrow S^{K} \rightarrow L(A) \hookrightarrow\left(\mathbb{G}_{m}\right)_{E / \mathbb{Q}} .
$$

The image of the middle homomorphism is $\operatorname{MT}(A)$.

A.6. Classification over $\mathbb{F}$ of abelian varieties. A Weil q-number $\left({ }^{8}\right)$ of weight $m$ is an element $\pi$ of a field of characteristic zero such that $q^{N} \pi$ is an algebraic integer for some $N$ and $\sigma(\pi) \cdot \iota(\sigma(\pi))=q^{m}$ for all homomorphisms $\sigma: \mathbb{Q}[\pi] \hookrightarrow \mathbb{C}$. The conditions imply that $q^{N} \pi$ is a unit at all finite primes $v$ of $\mathbb{Q}[\pi]$ not dividing $p$, and hence that the same is true for $\pi$. For any prime $v$ dividing $p$ of a field containing $\pi$, we let

$$
s_{\pi}(v)=\frac{\operatorname{ord}_{v}(\pi)}{\operatorname{ord}_{v}(q)}
$$

thus $s_{\pi}(v)+s_{\pi}(\iota v)=\operatorname{wt}(\pi)$. A Weil $q$-number is determined up to a root of 1 (as an element of an algebraic number field) by the numbers $s_{\pi}(v)$ because they determine all of its valuations. We call $s_{\pi}$ the slope function of $\pi$. A Weil $q$-number that is itself an integer is called a Weil q-integer.

Weil germs. Let $\pi$ be a Weil $p^{n}$-number and $\pi^{\prime}$ a Weil $p^{n^{\prime}}$-number in some field. We say $\pi$ and $\pi^{\prime}$ are equivalent if $\pi^{n^{\prime}}$ and $\pi^{\prime n}$ differ by a root of 1 . A Weil germ is an equivalence class of Weil numbers. The weight and slope function of a Weil germ $\pi$ are the weight and slope function of any representative of it, and $\mathbb{Q}[\pi]$ is defined to be the smallest field containing a representative of $\pi$. A Weil germ is determined by its slope function.

Let $W\left(p^{\infty}\right)$ denote the set of Weil germs represented by elements of $\mathbb{Q}^{\text {al }}$. It is an abelian group endowed with an action of $\Gamma$. Let $W\left(p^{\infty}\right)_{m,+}$ denote the subset of $W\left(p^{\infty}\right)$ consisting of Weil germs of weight $m$ represented by algebraic integers; thus,

$$
W\left(p^{\infty}\right)_{m,+}=\left\{\pi \in W\left(p^{\infty}\right) \mid s_{\pi}(v) \geq 0, s_{\pi}(v)+s_{\pi}(\iota v)=m \forall v\right\}
$$

Classification of abelian varieties. Let $A_{0}$ be a simple abelian variety over $\mathbb{F}$, and let $A_{1}$ be a model of $A_{0}$ over $\mathbb{F}_{q} \subset \mathbb{F}$ with the property that

$\left({ }^{8}\right)$ This conflicts with an earlier terminology (e.g., Tate 1968/69) which calls a "Weil $q$-number" what we call a "Weil $q$-integer of weight 1 ". 
$\operatorname{End}\left(A_{1}\right)=\operatorname{End}\left(A_{0}\right)$. The Frobenius endomorphism $\pi_{A_{1}}$ of $A_{1}$ is a Weil $q$-integer of weight 1 in $C\left(A_{0}\right)$, and we let $\pi_{A_{0}}$ denote the germ represented by $\pi_{A_{1}}$-it is independent of the choice of $A_{1} / \mathbb{F}_{q}$. The conjugates of $\pi_{A_{0}}$ in $\mathbb{Q}^{\text {al }}$ form a $\Gamma$-orbit $\Pi_{A_{0}}$ in $W\left(p^{\infty}\right)$, and the map $A_{0} \mapsto \Pi_{A_{0}}$ is a bijection from the set of isomorphism classes of simple abelian varieties over $\mathbb{F}$ onto the set of $\Gamma$-orbits in $W\left(p^{\infty}\right)_{1,+}$.

The various invariants of $A_{0}$ can be read off from $\Pi_{A_{0}}$ as follows. The images of $\mathbb{Q}\left[\pi_{A_{0}}\right]$ in $\mathbb{Q}^{\text {al }}$ are the fixed fields of the stabilizers of the different elements of $\Pi_{A_{0}}$, and so $\left[\mathbb{Q}\left[\pi_{A_{0}}\right]: \mathbb{Q}\right]=\left|\Pi_{A_{0}}\right|$. The division algebra $D={ }_{\mathrm{df}}$ $\operatorname{End}^{0}\left(A_{0}\right)$ has centre $\mathbb{Q}\left[\pi_{A_{0}}\right]$, and $D$ splits at no real prime of $\mathbb{Q}\left[\pi_{A_{0}}\right]$, splits at each finite prime not dividing $p$, and has invariant

$$
\operatorname{inv}_{v}(D)=s_{\pi}(v)\left[\mathbb{Q}\left[\pi_{A_{0}}\right]_{v}: \mathbb{Q}_{p}\right] \bmod \mathbb{Z},
$$

at each prime $v$ dividing $p$. By class field theory, the order of $D$ in the Brauer group of $\mathbb{Q}\left[\pi_{A_{0}}\right]$ is the smallest positive integer $e$ such that $e \cdot \operatorname{inv}_{v}(D) \in \mathbb{Z}$ for all $v$, and $\left[D: \mathbb{Q}\left[\pi_{A_{0}}\right]\right]^{1 / 2}=e$. Moreover,

$$
2 \operatorname{dim} A_{0}=\left[D: \mathbb{Q}\left[\pi_{A_{0}}\right]\right]^{1 / 2} \cdot\left[\mathbb{Q}\left[\pi_{A_{0}}\right]: \mathbb{Q}\right],
$$

and so $A_{0}$ has many endomorphisms. The set of slopes of the Dieudonné module of $A_{0}$ is $\left\{s_{\pi_{A_{0}}}(v)|v| p\right\}$, and an $s$ in this set has multiplicity

$$
\sum_{v, s_{\pi_{A_{0}}}(v)=s} \frac{2 \operatorname{dim} A_{0} \cdot\left[\mathbb{Q}\left[\pi_{A_{0}}\right]_{v}: \mathbb{Q}_{p}\right]}{\left[\mathbb{Q}\left[\pi_{A_{0}}\right]: \mathbb{Q}\right]} .
$$

It remains to classify the Weil germs.

Classification of Weil germs. Fix a CM-subfield $K$ of $\mathbb{Q}^{\text {al }}$, finite and Galois over $\mathbb{Q}$. For a Weil germ $\pi$ in $\mathbb{Q}^{\text {al }}$ and a prime $w$ of $\mathbb{Q}^{\text {al }}$ dividing $p$, let

$$
f_{\pi}^{K}(w)=s_{\pi}(w)\left[K_{w}: \mathbb{Q}_{p}\right] .
$$

Define $W^{K}\left(p^{\infty}\right)$ to be the set of Weil germs in $\mathbb{Q}^{\text {al }}$ represented by an element of $K$ and such that $f_{\pi}^{K}(w) \in \mathbb{Z}$. Since $W\left(p^{\infty}\right)=\bigcup_{K} W^{K}\left(p^{\infty}\right)$, it suffices to describe $W^{K}\left(p^{\infty}\right)$ for each $K$.

Let $F$ be the maximal real subfield of $K$, and let $X$ and $Y$ be the sets of primes in $K$ and $F$ respectively dividing $p$. Then there is an exact sequence

$$
0 \rightarrow W^{K}\left(p^{\infty}\right) \rightarrow \mathbb{Z}^{X} \times \mathbb{Z} \rightarrow \mathbb{Z}^{Y} \rightarrow 0 .
$$

The first map is $\pi \mapsto\left(f_{\pi}^{K}\right.$, wt $\left.(\pi)\right)$ and the second is

$$
(f, m) \mapsto f \mid Y-n_{0} \cdot m \cdot \sum_{v \in Y} v
$$

where $n_{0}=\left[K_{w}: \mathbb{Q}_{p}\right]$ for any prime $w$ of $K$ dividing $p$ (it is independent of $w$ ). Thus $\pi \mapsto f_{\pi}^{K}$ identifies $W^{K}\left(p^{\infty}\right)$ with the set of $f \in \mathbb{Z}^{X}$ such that $f(w)+f(\iota w)=n_{0} \cdot m$ for some integer $m$ (independent of $w$ ). 
Under $A_{0} \leftrightarrow \Pi_{A_{0}}$, the abelian varieties corresponding to orbits of $\Gamma$ in $W^{K}\left(p^{\infty}\right) \cap W\left(p^{\infty}\right)_{1,+}$ are those with the property that, for every $\sigma$ : $\mathbb{Q}\left[\pi_{A_{0}}\right] \hookrightarrow \mathbb{Q}^{\mathrm{al}}, \sigma \mathbb{Q}\left[\pi_{A_{0}}\right] \subset K$ and $\operatorname{End}^{0}\left(A_{0}\right) \otimes_{\mathbb{Q}\left[\pi_{A_{0}}\right], \sigma} K$ is a matrix algebra. Thus, there is a one-to-one correspondence between the isogeny classes of abelian varieties over $\mathbb{F}$ whose endomorphism algebra is split by $K$ in this sense and the $\operatorname{Gal}(K / \mathbb{Q})$-orbits of $f \in \mathbb{Z}^{X}$ such that $f(w)+f(\iota w)=n_{0}$ and $f(w) \geq 0$ for all $w$.

Remark. Given a possible slope function for a Weil germ $\pi$, the Dieudonné module of the corresponding abelian variety imposes restrictions on the possible factorizations of $p$ in $\mathbb{Q}[\pi]$. For example, suppose that $A(\pi)$ has slopes $0,1 / 2$, and 1 , that the multiplicity of $1 / 2$ is 2 , and that 0 and 1 do occur. Then the Dieudonné module of $A(\pi)$ has a simple isogeny factor of rank 2 , which implies that a prime $w$ for which $s_{\pi}(w)=1 / 2$ must be of degree 2 (if it had degree 1 , the action of $\mathbb{Q}[\pi]_{w}$ on the Dieudonné module would split off an isogeny factor of rank 1). Thus, the endomorphism algebra of such an abelian variety is commutative.

A.7. Calculation of the groups over $\mathbb{F}$. Let $A_{0}$ be an abelian variety over $\mathbb{F}$. Then $A_{0}$ is isogenous to a product $A_{1}^{s_{1}} \times \ldots \times A_{t}^{s_{t}}$ with the $A_{i}$ simple and pairwise nonisogenous, and $G\left(A_{0}\right) \cong G\left(A_{1} \times \ldots \times A_{t}\right)$ for $G=L, M$, or $P$; moreover, $L\left(A_{0}\right)_{0} \cong L\left(A_{1}\right)_{0} \times \ldots \times L\left(A_{t}\right)_{0}$. Thus, in the following, we assume that $A_{0}$ is a product of pairwise nonisogenous simple abelian varieties. Then $E={ }_{\mathrm{df}} C\left(A_{0}\right)$ is either a CM-algebra or the product of a CM-algebra with $\mathbb{Q}$ - the second case occurs when one of the isogeny factors of $A_{0}$ is a supersingular elliptic curve. The Rosati involution is complex conjugation on each CM-factor of $E$ and the identity map $\mathbb{Q}$.

The Lefschetz group. The description of $L\left(A_{0}\right)$ as a subgroup of $\left(\mathbb{G}_{m}\right)_{E / \mathbb{Q}}$ in terms of characters is the same as in the complex case.

Thus, the group $L\left(A_{0}\right)$ is the subgroup of $\left(\mathbb{G}_{m}\right)_{E / \mathbb{Q}}$ whose character group is

$$
\frac{\mathbb{Z}^{\Sigma_{E}}}{\left\{g \mid g=\iota g \text { and } \sum g(\sigma)=0\right\}} .
$$

The weight map $w: \mathbb{G}_{m} \rightarrow L\left(A_{0}\right)$ corresponds to the map

$$
[g] \mapsto \operatorname{wt}(g) \stackrel{\mathrm{df}}{=} \sum_{\sigma \in \Sigma_{E}} g(\sigma)
$$

on characters, and the homomorphism $t: L\left(A_{0}\right) \rightarrow \mathbb{G}_{m}$ giving the action of $L\left(A_{0}\right)$ on the Tate object sends 1 to the element of $X^{*}\left(L\left(A_{0}\right)\right)$ represented by $-\sigma-\iota \sigma$, any $\sigma \in \Sigma_{E}$.

It suffices to describe $L\left(A_{0}\right)_{0}$ in the case that $A_{0}$ is simple. When $A_{0}$ is a supersingular elliptic curve, $L\left(A_{0}\right)_{0}=\mu_{2}$; otherwise $L\left(A_{0}\right)_{0}$ is the subgroup 
of $\left(\mathbb{G}_{m}\right)_{E / \mathbb{Q}}$ whose character group is

$$
\frac{\mathbb{Z}^{\Sigma_{E}}}{\{g \mid g=\iota g\}} .
$$

The map $\mu_{2} \rightarrow L(A)_{0}$ corresponds to the map on characters $[g] \mapsto \sum g(\sigma)$ $\bmod 2$.

When $A_{0}$ is simple, the map $\sigma \mapsto \sigma\left(\pi_{A_{0}}\right): \Sigma_{E} \rightarrow \Pi_{A_{0}}$ is bijective and commutes with the action of $\Gamma$, and so identifies $L\left(A_{0}\right)$ with the torus whose character group is

$$
\frac{\mathbb{Z}^{\Pi_{A_{0}}}}{\left\{g \mid g=\iota g \text { and } \sum g(\pi)=0\right\}} .
$$

The group $P\left(A_{0}\right)$. By definition, $P\left(A_{0}\right) \subset L\left(A_{0}\right)$, and a character $[g]$ of $L\left(A_{0}\right)$ is trivial on $P\left(A_{0}\right)$ if and only if $g\left(\pi_{A_{0}}\right)=1$, where $g\left(\pi_{A_{0}}\right)$ is the Weil germ $\prod_{\sigma \in \Sigma_{E}}\left(\sigma \pi_{A_{0}}\right)^{g(\sigma)}$. A Weil germ is 1 if and only if its slopes are all zero, and so $[g]$ is trivial on $P\left(A_{0}\right)$ if and only if

$$
\sum_{\sigma \in \Sigma_{E}} g(\sigma) s_{\sigma \pi_{A_{0}}}(w)=0 \quad \text { for all } w \text {. }
$$

Note that $s_{\sigma \pi_{A_{0}}}(w)=s_{\pi_{A_{0}}}\left(\sigma^{-1} w\right)$. Similarly, a character $[g]$ of $L\left(A_{0}\right)_{0}$ is trivial on $L\left(A_{0}\right)_{0}$ if and only if $g\left(\pi_{A_{0}} / p^{1 / 2}\right)=g$ where $p^{1 / 2}$ also denotes the Weil germ represented by the Weil $p$-number $p^{1 / 2}$.

The motivic group. Fix a prime $\ell \in S\left(A_{0}\right)$ (see Appendix B). Let $\Omega_{\lambda}$ be a finite Galois extension of $\mathbb{Q}_{\ell}$ splitting $L\left(A_{0}\right)$, and let $\chi \in X^{*}\left(L\left(A_{0}\right)\right)$. Then $\chi$ is trivial on $M\left(A_{0}\right)$ if and only if $H^{2 r}\left(A_{0}^{s}, \Omega_{\lambda}(r)\right)_{\chi}$ contains a nonzero algebraic class for some $r$ and $s$, in which case all the spaces $H^{2 r}\left(A_{0}^{s}, \Omega_{\lambda}(r)\right)_{\chi}$ consist entirely of algebraic classes.

Second description of $P\left(A_{0}\right)$. Let $K$ be a CM-subfield of $\mathbb{Q}^{\text {al }}$, finite and Galois over $\mathbb{Q}$, and let $P^{K}$ be the torus over $\mathbb{Q}$ such that $X^{*}\left(P^{K}\right)=W^{K}\left(p^{\infty}\right)$ (as a $\Gamma$-module). Assume that $K$ is large enough to contain the conjugates of $\mathbb{Q}\left[\pi_{A_{0}}\right]$ and to split $\operatorname{End}^{0}\left(A_{0}\right)$. For any character $\chi$ of $L\left(A_{0}\right), \chi(\pi) \in$ $W^{K}\left(p^{\infty}\right)$. Thus we have a homomorphism $[g] \mapsto[g(\pi)]: X^{*}\left(L\left(A_{0}\right)\right) \rightarrow$ $W^{K}\left(p^{\infty}\right)$, which clearly commutes with the action of $\Gamma$. It corresponds to a homomorphism $\varrho_{A_{0}}: P^{K}\left(p^{\infty}\right) \rightarrow L\left(A_{0}\right)$, whose image is $P\left(A_{0}\right)$.

ExAMPLE. Let $A_{0}$ be isogenous to a product of elliptic curves, $A_{0} \sim$ $A_{1} \times \ldots \times A_{t}$, no two of which are isogenous. The centre $E$ of the endomorphism algebra of $A_{0}$ is the product $E=\prod E_{i}$ of the centres of the endomorphism algebras of the $A_{i}$. For each $i$, choose an embedding $\sigma_{i}: E_{i} \hookrightarrow \mathbb{Q}^{\text {al }}$. A character $g$ of $\left(\mathbb{G}_{m}\right)_{E / \mathbb{Q}}$ is trivial on $L\left(A_{0}\right)_{0}$ if and only if, for each $i$ for which $A_{i}$ is ordinary $g\left(\sigma_{i}\right)=g\left(\iota \sigma_{i}\right)$, and for each $i$ (there is at most one) for which $A_{i}$ is supersingular $2 \mid g\left(\sigma_{i}\right)$. 
Let $\pi \in E$ be a Weil $q$-number representing $\pi_{A_{0}}$; let $\pi=\left(\pi_{1}, \ldots, \pi_{t}\right)$. Then $g$ is trivial on $P\left(A_{0}\right)_{0}$ if and only if $g\left(\pi^{N}\right)=q^{N \cdot \operatorname{wt}(g) / 2}$ for some $N$. The statement (Spiess 1999, Proposition)

Let $\alpha_{1}, \ldots, \alpha_{2 m}$ be Weil $q$-numbers of elliptic curves over $\mathbb{F}_{q}$ such that $\alpha_{1} \ldots \alpha_{2 m}=q^{m}$; then, after possibly renumbering the $\alpha_{i}$ and replacing each $\alpha_{i}$ with $\alpha_{i}^{N}$ for some $N, \alpha_{2 j-1} \alpha_{2 j}=q$ for $j=1, \ldots, m$.

implies that this holds only if $g$ is trivial on $L\left(A_{0}\right)_{0}$. Thus $P\left(A_{0}\right)=L\left(A_{0}\right)$, and so no product of elliptic curves over $\mathbb{F}$ has an exotic Tate class.

EXAMPLE. Let $A_{0}$ be a simple abelian variety over $\mathbb{F}$ and let $\pi$ be its Frobenius germ. Assume that there is a prime $v_{1}$ of degree 1 of $\mathbb{Q}[\pi]$ such that $s_{\pi}\left(v_{1}\right)=0, s_{\pi}\left(\iota v_{1}\right)=1$, and $s_{\pi}(v)=1 / 2$ for $v \neq v_{1}$. Let $\pi_{1}$ be a Weil

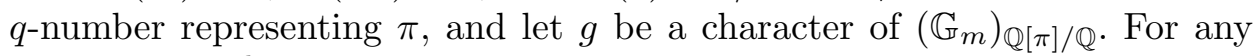
prime $w$ of $\mathbb{Q}^{\text {al }}$ dividing $p$,

$$
\operatorname{ord}_{w}\left(g\left(\pi_{1} / q^{1 / 2}\right)\right)=\frac{\operatorname{ord}_{v_{1}} q}{2}(-g(\sigma)+g(\iota \sigma))
$$

where $\sigma$ is the unique embedding of $\mathbb{Q}[\pi]$ such that $\sigma^{-1} w=v_{1}$. Therefore, $g$ is trivial on $P\left(A_{0}\right)_{0}$ if and only if $g=\iota g$, i.e., if and only if $g$ is trivial on $L\left(A_{0}\right)_{0}$. Thus $P\left(A_{0}\right)=L\left(A_{0}\right)$, and no power of $A_{0}$ has an exotic Tate class. In particular, the Tate conjecture holds for the powers of $A_{0}$.

The abelian varieties of "K3-type" of Zarhin 1993 are covered by this example (they are the varieties for which, additionally, $[\mathbb{Q}[\pi]: \mathbb{Q}]=2 \operatorname{dim} A_{0}$ ).

ExAMPLE. Let $A_{0}$ be a simple abelian variety of dimension $>1$ over $\mathbb{F}$ and let $\pi$ be its Frobenius germ. Assume that there is a prime $v_{1}$ of $\mathbb{Q}[\pi]$ whose decomposition group is $\{1, \iota\}$ for which $s_{\pi}\left(v_{1}\right)=1 / 2=s_{\pi}\left(\iota v_{1}\right)$; assume moreover that $s_{\pi}(v)=0$ or 1 for all other primes. Let $\pi_{1}$ be a Weil $q$-number representing $\pi$, and let $\chi$ be a character of $X^{*}\left(L\left(A_{0}\right)_{0}\right)$ that is trivial on $P\left(A_{0}\right)_{0}$. If $\chi=m \chi_{1}$ for some $\chi_{1} \in X^{*}\left(L\left(A_{0}\right)_{0}\right)$, then $\chi_{1}$ is also trivial on $P\left(A_{0}\right)_{0}$. Thus, we may assume that $\chi$ is not divisible in $X^{*}\left(L\left(A_{0}\right)_{0}\right)$. Let $g=\sum g(\sigma) \sigma$ be an element of $\mathbb{Z}^{\Sigma_{\mathbb{Q}[\pi]}}$ representing $\chi$ and such that $g(\sigma) \neq 0 \Rightarrow g(\iota \sigma)=0$. For any prime $w$ of $\mathbb{Q}^{\text {al }}$ dividing $p$,

$$
\operatorname{ord}_{w}\left(g\left(\pi_{1}\right)\right) / \operatorname{ord}_{w}(q) \equiv \frac{1}{2} g(\sigma) \bmod \mathbb{Z}
$$

where $\sigma$ is such that $\sigma^{-1} w=v_{1}$. Hence $g(\sigma)$ is even. As $w$ ranges over the primes dividing $p, \sigma$ ranges over the elements of $\Sigma_{\mathbb{Q}\left[\pi_{A_{0}}\right]}$ for which $g(\sigma) \neq 0$. This contradicts the fact that $\chi$ is not divisible. Hence $\chi=0$, and we see that $P\left(A_{0}\right)=L\left(A_{0}\right)$. Hence no power of $A_{0}$ has an exotic Tate class.

The "almost ordinary" abelian varieties of Lenstra and Zarhin 1993 are covered by this example.

A.8. Reduction of abelian varieties with many endomorphisms: the fundamental diagram. Fix a prime $w_{0}$ of $\mathbb{Q}^{\text {al }}$ dividing $p$, and let $\mathbb{F}$ 
be the residue field. As we noted in Section 1, it follows from the theory of Néron models that an abelian variety $A$ over $\mathbb{Q}^{\text {al }}$ with many endomorphisms has good reduction at $w_{0}$ to an abelian variety $A_{0}$ over $\mathbb{F}$. We shall explain the map $A \mapsto A_{0}$ in terms of the above classifications.

Assume $A$ is isotypic, and let $E$ be a CM-subfield of $\operatorname{End}^{0}(A)$ for which $H^{1}(A, \mathbb{Q})$ is free of rank 1 , and let $\Phi$ be the CM-type on $E$ defined by its action on $H^{1,0}$. Let $\pi_{A_{0}}$ be the Weil germ of $A_{0}$ in $E$. We fix an embedding $\varrho_{0}: E \hookrightarrow \mathbb{Q}^{\text {al }}$, and explain how to construct $\varrho_{0}\left(\pi_{A_{0}}\right)$. Let $K$ be a CM-subfield of $\mathbb{Q}^{\text {al }}$, finite and Galois over $\mathbb{Q}$, and large enough to contain all conjugates of $E$. As a subfield of $\mathbb{Q}^{\text {al }}, K$ acquires a prime $w_{0}$. For some $h, \mathfrak{P}_{w_{0}}^{h}$ will be principal, say $\mathfrak{P}_{w_{0}}^{h}=(a)$. Let $\alpha=a^{2 n}$ where $n$ is the index of the unit group of the maximal real subfield of $K$ in the full unit group of $K$. Then $\psi_{\varrho_{0}}(\alpha)$, where $\psi_{\varrho_{0}}$ is the CM-type on $K$ defined in A.4, is a well-defined Weil $p^{2 n h f\left(\mathfrak{P}_{w_{0}} / p\right)}$-integer of weight 1 lying in $\varrho_{0} E$. Its inverse image in $E$ represents $\pi_{A_{0}}$.

Assume now that $E$ is a field. The value of the function $s_{\pi_{A_{0}}}$ on a prime $v$ of $E$ dividing $p$ is given by

where

$$
s_{\pi_{A_{0}}}(v)=\frac{|\Phi(v)|}{\left|\Sigma_{E}(v)\right|}
$$

$$
\Sigma_{E}(v)=\left\{\sigma \in \Sigma_{E} \mid v=\sigma^{-1} w_{0}\right\}, \quad \Phi(v)=\Phi \cap \Sigma_{E}(v) .
$$

Suppose $A$ is simple, and that it corresponds to a $\operatorname{Gal}(K / \mathbb{Q})$-orbit $\Psi$ in $X^{*}\left(S^{K}\right)$. An element $f \in X^{*}\left(S^{K}\right)$ can be regarded as a function $f: \Sigma_{K} \rightarrow$ $\mathbb{Z}$. Define $\bar{f}$ to be the function $X \rightarrow \mathbb{Z}$ such that $\bar{f}(w)=\sum_{\tau w_{0}=w} f(\tau)$, i.e., if $f$ is $\sum f(\tau) \tau$, then $\bar{f}$ is $\sum f(\tau) \tau w_{0}$. Then $A_{0}$ is isogenous to a power of a simple abelian variety, which corresponds (as in A.6) to the $\operatorname{Gal}(K / \mathbb{Q})$-orbit $\{\bar{f} \mid f \in \Psi\} \subset W^{K}\left(p^{\infty}\right)$.

Let $K$ be a CM-field, finite and Galois over $\mathbb{Q}$, and let $F$ be the maximal totally real subfield of $K$. If no $p$-adic prime of $F$ splits in $K$, then $S^{K}=\mathbb{G}_{m}$ and the only elements of $W^{K}\left(p^{\infty}\right)$ are those represented by the Weil $p$ numbers $p^{m / 2}$. Otherwise, all the $p$-adic primes in $F$ split in $K$, and there is an exact commutative diagram:

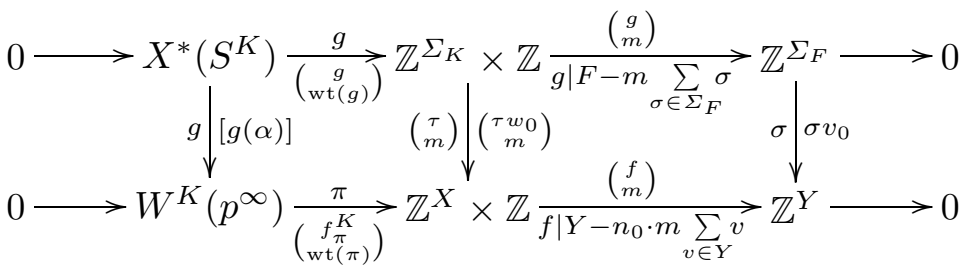

The element above (or to the left of) an arrow is mapped to the element below (or to the right) by the arrow. The symbol $g(\alpha)$ denotes $\prod \sigma(\alpha)^{g(\sigma)}$, $n_{0}=\left[K_{w_{0}}: \mathbb{Q}_{p}\right]$, and $v_{0}$ is the prime on $F$ induced by $w_{0}$. 
We saw above that an abelian variety $(A, i)$ of CM-type $(E, \Phi)$ reduces modulo the prime $w_{0}$ of $\mathbb{Q}^{\text {al }}$ to an isotypic abelian variety $A_{0}$ whose Weil germ is determined by $(* * *)$. Every simple abelian variety arises in this way: let $A_{0}$ be a simple abelian variety over $\mathbb{F}$, and let $E$ be a CM-field that can be embedded as a maximal subfield of $\operatorname{End}^{0}(A)$ containing $\mathbb{Q}\left[\pi_{A_{0}}\right]$; algebraic number theory shows that $E$ exists, and it is an elementary exercise to show that there exist CM-types $\Phi$ on $E$ such that $s_{\pi_{A_{0}}}$ is given by the formula (***); let $A$ be an abelian variety over $\mathbb{Q}^{\text {al }}$ of CM-type $(E, \Phi)$; it is uniquely determined up to isogeny, and $A_{0}$ is isogenous to the reduction of $A$ at $w_{0}$.

Thus, to give a lifting (up to isogeny) of $A_{0}$ to characteristic zero is to give a CM maximal subfield $E$ of $\operatorname{End}^{0}(A)$ and a CM-type on $E$ satisfying (***).

\section{Appendix B. Numerical equivalence on abelian varieties with many endomorphisms}

Let $A$ be an abelian variety of dimension $g$ over an algebraically closed field. In characteristic zero, two cycles in $\mathcal{Z}^{r}(A)$ are homologically equivalent if their classes in $H^{2 r}(A, \mathbb{Q}(r))$ are equal, and in characteristic $p \neq 0$, they are $\ell$-homologically equivalent, $\ell \neq p$, if their classes in the étale cohomology group $H^{2 r}\left(A, \mathbb{Q}_{\ell}(r)\right)$ are equal. Because of Poincaré duality and the compatibility of intersection products with cup products, homological equivalence implies numerical equivalence. It is generally conjectured that they coincide.

Part (a) of the following theorem is a special case of a theorem of Lieberman (1968, Theorem 4), and part (b) is a theorem of Clozel (1999). The proof is based on that of Clozel.

Theorem B.1. (a) For any abelian variety $A$ with many endomorphisms over an algebraically closed field $k$ of characteristic zero, homological equivalence coincides with numerical equivalence on $\mathcal{Z}^{r}(A)$, all $r$.

(b) For any abelian variety $A_{0}$ over $\mathbb{F}$, there exists a set $S$ of primes $\ell$ of density $>0$ (depending on $A_{0}$ ) for which $\ell$-homological equivalence coincides with numerical equivalence on $\mathcal{Z}^{r}\left(A_{0}\right)$, all $r$.

Proof. In the proof, we ignore Tate twists, i.e., we choose an identification of $\mathbb{Q} \approx \mathbb{Q}(1)\left(\right.$ or $\left.\mathbb{Q}_{\ell} \approx \mathbb{Q}_{\ell}(1)\right)$.

First consider the characteristic zero case. Choose $\left({ }^{9}\right)$ an étale CMalgebra $E \subset \operatorname{End}^{0}(A)$ such that $H^{1}(A, \mathbb{Q})$ is free of rank 1 as an $E$-module and $E$ is stable under the Rosati involution defined by some ample divi-

$\left({ }^{9}\right)$ For each isotypic isogeny factor $A_{i}$ of $A$, choose a CM-field $E_{i}$ in $\operatorname{End}^{0}\left(A_{i}\right)$ of degree $2 \operatorname{dim} A_{i}$, and let $E=\prod E_{i}$. Write $H_{1}(A, \mathbb{Q})=E \cdot x_{0}$. For any $c \in E^{\times}$such that $\iota_{E} c=-c, a x_{0}, b x_{0} \mapsto \operatorname{Tr}_{E / \mathbb{Q}}(c a b)$ is a Riemann form on $A$, and we can take $D$ to be any divisor whose class it is. When $A, E$, and $\Phi$ are as in this paragraph, one says that $(A, i)$, where $i$ is the inclusion $E \hookrightarrow \operatorname{End}^{0}(A)$, is of $C M$-type $(E, \Phi)$. 
sor $D$. The action of $E \otimes_{\mathbb{Q}} \mathbb{R}$ on $H^{1,0}$ defines a CM-type $\Phi$ on $E$. We have $\operatorname{Hom}\left(E, \mathbb{Q}^{\mathrm{al}}\right)=\Phi \sqcup \bar{\Phi}$.

Let $\Omega$ be the smallest subfield of $\mathbb{Q}^{\text {al }}$ containing $\sigma E$ for every homomorphism $\sigma: E \rightarrow \mathbb{Q}^{\text {al }}$. It is a CM-field, finite and Galois over $\mathbb{Q}$. Let $H^{r}(A, \mathbb{Q})_{\Omega}=H^{r}(A, \mathbb{Q}) \otimes \Omega$, and let $H^{1}(A)_{\sigma}$ be the subspace of $H^{1}(A, \mathbb{Q})_{\Omega}$ on which $E$ acts through $\sigma$. Then $H^{1}(A, \mathbb{Q})_{\Omega}=\bigoplus_{\sigma \in \Phi \sqcup \bar{\Phi}} H^{1}(A)_{\sigma}$ and $H^{1}(A)_{\sigma}$ is one-dimensional. As $H^{r}(A, \mathbb{Q})_{\Omega}=\bigwedge_{\Omega}^{r} H^{1}(A, \mathbb{Q})_{\Omega}$, it follows that

$$
H^{r}(A, \mathbb{Q})_{\Omega}=\bigoplus_{I, J,|I|+|J|=r} H^{r}(A)_{I, J}
$$

where $I$ and $J$ are subsets of $\Phi$ and $\iota \Phi$ respectively, and $H^{r}(A)_{I, J}=_{\mathrm{df}}$ $H^{r}(A)_{I \sqcup J}$ is the subspace on which $e \in E$ acts as $\prod_{\sigma \in I \sqcup J} \sigma e$-it is of dimension 1 and of Hodge type $(|I|,|J|)$. For $x \in H^{r}(A, \mathbb{Q})_{\Omega}$, let $x_{I, J}$ denote the projection of $x$ on $H^{r}(A)_{I, J}$. Because $x \mapsto x_{I, J}$ is multiplication by an idempotent $e_{I, J}$ of $E \otimes \Omega$, it sends algebraic classes to algebraic classes.

Let $L$ be the class in $H^{2}(A, \mathbb{Q})$ of the divisor $D$. Because $L$ is algebraic, its isotypic components in $H^{2}(A, \mathbb{Q})_{\Omega}$ are of type $(\sigma, \iota \sigma), \sigma \in \Sigma_{E}$, and, because $L$ defines a nondegenerate form on $H_{1}(A, \mathbb{Q})$, each such component is nonzero.

For each $\sigma$, choose a nonzero element $\omega_{\sigma}$ of $H^{1}(A)_{\sigma}$. Then $\left(\omega_{\sigma}\right)_{\Phi \sqcup \iota \Phi}$ is a basis for $H^{1}(A, \mathbb{Q})_{\Omega}$. We may suppose that the $\omega_{\sigma}$ have been chosen so that the $(\sigma, \iota \sigma)$ component of $L$ is $\omega_{\sigma} \omega_{\iota \sigma}$. Denote $\prod_{\sigma \in I} \omega_{\sigma} \prod_{\sigma \in J} \omega_{\sigma}$ by $\omega_{I, J}-$ it is a basis for $H^{r}(A)_{I, J}$. For $i \leq g=\operatorname{dim} A$,

$$
L^{g-i}=\left(\sum_{\sigma \in \Sigma_{E}} \omega_{\sigma} \omega_{\iota \sigma}\right)^{g-i}=\sum_{M}(g-i) ! \omega_{M, \iota M}
$$

where $M$ runs over the subsets of $\Phi$ with $|M|=g-i$. In particular, $\omega_{M, \iota M}$ is algebraic. Moreover,

$$
L^{g-i} \omega_{I J}=\sum_{|M|=g-i}(g-i) ! \omega_{I \cup M, J \cup \iota M} .
$$

Only the subsets $M$ disjoint from both $I$ and $J$ contribute to the sum.

We shall need the following theorem of Lieberman (Kleiman 1968, 2A11 and 2.2):

Let $\mathcal{A}^{r}$ be the space of algebraic classes in $H^{2 r}(A, \mathbb{Q})$; then for $2 r \leq g$, the map $L^{g-2 r}: \mathcal{A}^{r} \rightarrow \mathcal{A}^{g-r}$ is an isomorphism.

Suppose $\omega_{I J}$ is algebraic with $|I|+|J|=2 r \leq g$. Let $M=I \cap \iota J$, so that there exist $I_{0}$ and $J_{0}$ for which $I=I_{0} \sqcup M, J=J_{0} \sqcup \iota M, I_{0} \cap \iota J_{0}=\emptyset$. We shall prove by induction on $|I \cap \iota J|$ that $\omega_{I_{0}, J_{0}}$ is also algebraic. If $|I \cap \iota J|=0$, there is nothing to prove. If not, $|I \cup \iota J| \leq 2 r-1$, and there exists a subset $M$ of $\Phi$ with $g-2 r+1$ elements disjoint from $I \cup \iota J$. Then $\omega_{I \sqcup M, J \sqcup \iota M}$ is nonzero 
and algebraic. By Lieberman's theorem, there exists an $x \in \mathcal{A}^{r-1}$ such that $L^{g-2 r+2} x=\omega_{I \sqcup M, J \sqcup \iota M}$. If $\omega_{I^{\prime}, J^{\prime}}$ occurs with nonzero coefficient in $x$, then it is algebraic. But if $\omega_{I^{\prime}, J^{\prime}}$ is chosen so that $\omega_{I \sqcup M, J \sqcup \iota M}$ occurs with nonzero coefficient in $L^{g-2 r+2} \omega_{I^{\prime}, J^{\prime}}$, then $I_{0}^{\prime}=I_{0}, J_{0}^{\prime}=J_{0}$. Since $\left|I^{\prime} \cap \iota J^{\prime}\right|=|I \cap J|-2$, the induction hypothesis shows that $\omega_{I_{0}, J_{0}}$ is algebraic.

We now prove the theorem in the case of characteristic zero. We have to show that, for each $r \leq g$, the cup-product pairing

$$
\mathcal{A}^{r} \times \mathcal{A}^{g-r} \rightarrow \mathbb{Q}
$$

is nondegenerate. Lieberman's theorem shows that the two spaces have the same dimension, and so it suffices to show that the left kernel is zero. Thus, let $x$ be a nonzero element of $\mathcal{A}^{r}, r \leq g$, and suppose $\omega_{I, J}$ occurs with nonzero coefficient in $x$. It suffices to show that $\omega_{I^{\prime}, J^{\prime}}$ is algebraic, where $I^{\prime}$ and $J^{\prime}$ are the complements of $I$ and $J$ in $\Phi$ and $\iota \Phi$ respectively. From the last paragraph, we know that $\omega_{I, J}=\omega_{I_{0} \sqcup M, J_{0} \sqcup \iota M}$ with $\omega_{I_{0}, J_{0}}$ algebraic and $I_{0}, \iota J_{0}$, and $M$ disjoint. Because $\mathcal{A}^{j} \otimes_{\mathbb{Q}} \Omega$ is stable under $\operatorname{Gal}(\Omega / \mathbb{Q})$, $\iota \omega_{I_{0}, J_{0}}=\omega_{\iota J_{0}, \iota I_{0}}$ is algebraic. But $\omega_{I^{\prime}, J^{\prime}}=\omega_{\iota J_{0}, \iota I_{0}} \cdot \omega_{N, \iota N}$ where $N$ is the complement of $I_{0} \sqcup \iota J_{0} \sqcup M$ in $\Phi$, which is obviously algebraic.

We now prove the theorem in the case $k=\mathbb{F}$. After possibly replacing $A_{0}$ with an isogenous variety, we may assume that it lifts to an abelian variety $A$ with many endomorphisms in characteristic zero (see A.8). Let $E$ be a CM-algebra for $A$ as in the first paragraph of the proof. If $\ell$ is such that $\iota$ is in the decomposition group of some prime $\lambda$ of $\Omega$ dividing $\ell$, then the same argument as in characteristic zero case applies once one replaces $\mathbb{Q}$ with $\mathbb{Q}_{\ell}$ and $\Omega$ with $\Omega_{\lambda}$ (Lieberman's theorem holds for every Weil cohomology; in particular, it holds for the étale cohomology). The Frobenius density theorem shows that the set of primes $\ell$ such that $\iota$ is the Frobenius element at a prime $\lambda$ dividing $\ell$ has density $1 /[\Omega: \mathbb{Q}]$. For such a prime $\ell, \iota$ is in the decomposition group of $\lambda$.

Let $A_{0}$ be an abelian variety over $\mathbb{F}$, and let $E_{0}$ be the centre $C\left(A_{0}\right)$ of $\operatorname{End}^{0}\left(A_{0}\right)$. When $A_{0}$ does not have a supersingular elliptic curve as an isogeny factor, we define $\Omega_{0}$ to be the composite of the fields $\sigma E_{0}$ for $\sigma \in$ $\Sigma_{E_{0}}$; otherwise we define it to be the composite of these fields with $\mathbb{Q}[\sqrt{-p}]$. Define $S\left(A_{0}\right)$ to be the set of finite primes $\ell \neq p$ such that $\iota$ is contained in the decomposition group of $\lambda$ for one (hence every) prime $\lambda$ of $\Omega_{0}$ dividing $\ell$. Note that $S\left(A_{0}\right)$ depends only on the finite set of simple isogeny factors of $A_{0}$; in particular, $S\left(A_{0}\right)=S\left(A_{0}^{s}\right)$.

Proposition B.2. Statement (b) of Theorem B.1 holds with $S=S\left(A_{0}\right)$.

Proof. Suppose $A_{0}$ is isogenous to $A_{1}^{s_{1}} \times \ldots \times A_{t}^{s_{t}}$ with the $A_{i}$ simple and nonisogenous in pairs. Assume initially that none of the $A_{i}$ is a supersingular elliptic curve. Then each $C\left(A_{i}\right)$ is a CM-field. 
For each $i$, let $D_{i}=\operatorname{End}^{0}\left(A_{i}\right)$, let $m_{i}=\left[D_{i}: C\left(A_{i}\right)\right]^{1 / 2}$, and let $C\left(A_{i}\right)_{+}$ be the maximal real subfield of $C\left(A_{i}\right)$. Fix an $\ell \in S\left(A_{0}\right)$. For each $i$, there exists a field $F_{i}$ cyclic of degree $m_{i}$ over $C\left(A_{i}\right)_{+}$and such that each real and $\ell$-adic prime of $C\left(A_{i}\right)_{+}$splits in $F_{i}$ and the local degree at each $p$-adic prime is $m_{i}$ (Artin and Tate 1961, p. 105, Theorem 5). Let $E_{i}=F_{i} \cdot C\left(A_{i}\right)$. Then $E_{i}$ is a CM-field that splits $D_{i}$ (Tate $1968 / 69$, p. 7 ) and can be realized as a subfield of $D_{i}$. Therefore (Tate 1968/69, Théorème 2), $A_{i}$ is isogenous to the reduction of an abelian variety $\widetilde{A}_{i}$ with $\operatorname{End}^{0}\left(\widetilde{A}_{i}\right)=E_{i}$.

After replacing $A_{0}$ with an isogenous variety, we may suppose that it lifts to the abelian variety $A={ }_{\mathrm{df}} \widetilde{A}_{1}^{s_{1}} \times \ldots \times \widetilde{A}_{t}^{s_{t}}$. The étale algebra $E={ }_{\mathrm{df}}$ $E_{1}^{s_{1}} \times \ldots \times E_{t}^{s_{t}}$ acts on $A$ diagonally, and satisfies the conditions in the first paragraph of the proof of Theorem B.1. The field $\Omega$ generated by the images of $E$ in $\mathbb{Q}^{\text {al }}$ is $\Omega_{0} \cdot F_{1} \ldots F_{t}$. Because of our choice of the $F_{i}$, every $\ell$-adic prime in this field is fixed $\left({ }^{10}\right)$ by $\iota$. This completes the proof of the proposition in this case.

When we add a factor $A_{t+1}^{s_{t+1}}$ to $A_{0}$ with $A_{t+1}$ a supersingular elliptic curve, $\Omega$ is replaced with $\Omega \cdot E_{t+1}$ where $E_{t+1}$ can be taken to be any quadratic field in which $p$ does not split. If we choose $E_{t+1}=\mathbb{Q}[\sqrt{-p}]$, then $\Omega=\Omega_{0} \cdot F_{1} \ldots F_{t}$ still holds, and the same argument applies.

Let $A$ be an abelian variety with many endomorphisms over $\mathbb{Q}^{\text {al }}$, and let $A_{0}$ be its reduction at the prime $w_{0}$. Fix an $\ell \neq p$. Then there are canonical isomorphisms $H^{i}\left(A, \mathbb{Q}_{\ell}(j)\right) \rightarrow H^{i}\left(A_{0}, \mathbb{Q}_{\ell}(j)\right)$ for all $i$ and $j$ (proper and smooth base change theorems in étale cohomology). We say that a cohomology class $\gamma \in H^{2 r}(A, \mathbb{Q}(r))$ is $w_{0}$-algebraic if its image $\gamma_{\ell}$ in $H^{2 r}\left(A_{0}, \mathbb{Q}_{\ell}(r)\right)$ is in the $\mathbb{Q}$-span of the algebraic classes on $A_{0}$. Every algebraic class is $w_{0}$-algebraic, but not every $w_{0}$-algebraic class is algebraic.

Theorem B.3. For any nonzero $w_{0}$-algebraic class $\alpha$ on $A$, there exists a $w_{0}$-algebraic class $\alpha^{\prime}$ such that $\alpha \cup \alpha^{\prime} \neq 0$.

Proof. Let $\mathcal{A}^{r}\left(w_{0}\right)$ be the $\mathbb{Q}$-space of $w_{0}$-algebraic classes in $H^{2 r}(A, \mathbb{Q}(r))$. The proof of the characteristic zero case of the Theorem in A.3 will apply with "algebraic" replaced by " $w_{0}$-algebraic" once we have shown that Lieberman's theorem holds for $\mathcal{A}^{r}\left(w_{0}\right)$ : for $2 r \leq g, L^{g-2 r}: \mathcal{A}^{r}\left(w_{0}\right) \rightarrow \mathcal{A}^{g-r}\left(w_{0}\right)$ is an isomorphism.

This map is automatically injective, and so we only have to prove surjectivity.

Let $\gamma$ be a $w_{0}$-algebraic class in $H^{2 g-2 r}(A, \mathbb{Q}(g-r))$; by assumption, the image $\gamma_{\ell}$ of $\gamma$ in $H^{2 g-2 r}\left(A_{0}, \mathbb{Q}_{\ell}(g-r)\right)$ equals the class $\alpha_{\ell}$ of some

$\left({ }^{10}\right)$ Let $F_{0}$ be the maximal totally real subfield of $\Omega_{0}$. The condition that $\iota$ fixes all $\ell$-adic primes in $\Omega_{0}$ means that, for each $\ell$-adic prime $v$ of $F_{0}, \Omega_{0} \otimes_{F_{0}}\left(F_{0}\right)_{v}$ is a field. Because $\ell$ splits in $F_{1} \ldots F_{t}$, this property is retained by $\Omega$. 
$\alpha \in \mathcal{Z}^{g-r}\left(A_{0}\right)$. There exists a $\gamma^{\prime} \in H^{2 r}(A, \mathbb{Q}(r))$ such that $L^{g-2 r} \gamma^{\prime}=$ $\gamma$ (hard Lefschetz theorem), and Lieberman's theorem says that there is an $\alpha^{\prime} \in \mathcal{Z}^{r}\left(A_{0}\right)$ such that $L^{g-2 r} \alpha^{\prime}=\alpha$. The images of $\alpha^{\prime}$ and $\gamma^{\prime}$ in $H^{2 r}\left(A_{0}, \mathbb{Q}_{\ell}(r)\right)$ map to $\alpha_{\ell}$ and $\gamma_{\ell}$ respectively under the isomorphism $L^{g-r}$ : $H^{2 r}\left(A_{0}, \mathbb{Q}_{\ell}(r)\right) \rightarrow H^{2 g-2 r}\left(A_{0}, \mathbb{Q}_{\ell}(g-r)\right)$. As $\alpha_{\ell}=\gamma_{\ell}$, this proves that $\gamma^{\prime}$ is $w_{0}$-algebraic.

Corollary B.4. Suppose that the $\ell$-adic cohomology class $c_{\ell}$ of $c \in$ $\mathcal{Z}^{r}\left(A_{0}\right)$ is nonzero. If $c_{\ell}$ is the image of a rational cohomology class on $A$ (i.e., of an element of $H^{2 r}(A, \mathbb{Q}(r))$ ), then $c$ is not numerically equivalent to zero.

Proof. Immediate consequence of the theorem and the compatibility of the cup-product pairings.

The corollary implies that, if every algebraic class on $A_{0}$ "lifts" to a rational cohomology class in characteristic zero, then $\ell$-adic homological equivalence on $A_{0}$ coincides with numerical equivalence.

\section{References}

E. Artin and J. Tate (1961), Class Field Theory, Harvard, Dept. of Mathematics.

L. Clozel (1999), Équivalence numérique et équivalence cohomologique pour les variétés abéliennes sur les corps finis, Ann. of Math. 150, 151-163.

P. Deligne (1982), Hodge cycles on abelian varieties (notes by J. S. Milne), in: Hodge Cycles, Motives, and Shimura Varieties, Lecture Notes in Math. 900, Springer, Heidelberg, 9-100.

P. Deligne and J. S. Milne (1982), Tannakian categories, in: Hodge Cycles, Motives, and Shimura Varieties, Lecture Notes in Math. 900, Springer, Heidelberg, 101-228.

B. van Geemen (1994), An introduction to the Hodge conjecture for abelian varieties, in: Algebraic Cycles and Hodge Theory (Torino, 1993), Lecture Notes in Math. 1594, Springer, Berlin, 233-252.

B. van Geemen (1996), Theta functions and cycles on some abelian fourfolds, Math. Z. $221,617-631$.

U. Jannsen (1992), Motives, numerical equivalence, and semisimplicity, Invent. Math. 107, $447-459$.

S. Kleiman (1968), Algebraic cycles and the Weil conjectures, in: Dix Exposés sur la Cohomologie des Schémas, North-Holland, Amsterdam, 359-386.

H. Lange and Ch. Birkenhake (1992), Complex Abelian Varieties, Grundlehren Math. Wiss. 302, Springer, Berlin.

H. W. Lenstra and Y. G. Zarhin (1993), The Tate conjecture for almost ordinary abelian varieties over finite fields, in: Advances in Number Theory (Kingston, ON, 1991), Oxford Sci. Publ., Oxford Univ. Press, New York, 179-194.

D. I. Lieberman (1968), Numerical and homological equivalence of algebraic cycles on Hodge manifolds, Amer. J. Math. 90, 366-374.

J. S. Milne (1999a), Lefschetz classes on abelian varieties, Duke Math. J. 96, 639-675. 
J. S. Milne (1999b), Lefschetz motives and the Tate conjecture, Compositio Math. 117, $47-81$

F. Oort (1973), The isogeny class of a CM-type abelian variety is defined over a finite extension of the prime field, J. Pure Appl. Algebra 3, 399-408.

H. Pohlmann (1968), Algebraic cycles on abelian varieties of complex multiplication type, Ann. of Math. (2) 88, 161-180.

N. Saavedra Rivano (1972), Catégories Tannakiennes, Lecture Notes in Math. 265, Springer, Berlin.

C. Schoen (1988), Hodge classes on self-products of a variety with an automorphism, Compositio Math. 65, 3-32.

C. Schoen (1998), Addendum to: "Hodge classes on self-products of a variety with an automorphism", ibid. 114, 329-336.

J.-P. Serre and J. Tate (1968), Good reduction of abelian varieties, Ann. of Math. (2) 88, $492-517$.

M. Spiess (1999), Proof of the Tate conjecture for products of elliptic curves over finite fields, Math. Ann. 314, 285-290.

J. Tate (1966), Endomorphisms of abelian varieties over finite fields, Invent. Math. 2, $134-144$.

J. Tate (1968/69), Classes d'isogénie des variétés abéliennes sur un corps fini (d'après T. Honda), Séminaire Bourbaki, 21e année no. 352.

J. Tate (1994), Conjectures on algebraic cycles in l-adic cohomology, in: Motives (Seattle, WA, 1991), Proc. Sympos. Pure Math. 55, Part 1, Amer. Math. Soc., Providence, RI, 71-83.

A. Weil (1977), Abelian varieties and the Hodge ring, CEuvres Scientifiques, Vol. III, 421429.

Y. G. Zarhin (1993), Abelian varieties of K3 type, in: Séminaire de Théorie des Nombres, Paris, 1990-91, Progr. Math. 108, Birkhäuser, Boston, MA, 263-279.

2679 Bedford Rd

Ann Arbor, MI 48104, U.S.A.

E-mail: math@jmilne.org

URL: www.jmilne.org/math/

Received on 19.8.1999

and in revised form on 1.3.2001 